\title{
EMPIRICAL PREDICTIONS FOR (SUB-)MILLIMETER LINE AND CONTINUUM DEEP FIELDS
}

\author{
Elisabete da Cunha ${ }^{1}$, Fabian Walter $^{1}$, Roberto Decarli ${ }^{1}$, Frank Bertoldi $^{2}$, Chris Carilli $^{3}$, Emanuele Daddi ${ }^{4}$, \\ David Elbaz $^{4}$, Rob Ivison ${ }^{5,6}$, Roberto Maiolino ${ }^{7,8}$, Dominik Riechers ${ }^{9,10}$, Hans-Walter Rix ${ }^{1}$, Mark SARgent ${ }^{4}$, \\ IAN SMAIL ${ }^{11}$, AND AXeL WeIss ${ }^{12}$ \\ ${ }^{1}$ Max-Planck-Institut für Astronomie, Königstuhl 17, D-69117 Heidelberg, Germany; cunha@mpia.de \\ ${ }^{2}$ Argelander Institute for Astronomy, University of Bonn, Auf dem Hügel 71, D-53121 Bonn, Germany \\ ${ }^{3}$ National Radio Astronomy Observatory, Pete V. Domenici Array Science Center, P.O. Box O, Socorro, NM 87801, USA \\ ${ }^{4}$ Laboratoire AIM, CEA/DSM-CNRS-Université Paris Diderot, Irfu/Service d'Astrophysique, CEA Saclay, Orme des Merisiers, \\ F-91191 Gif-sur-Yvette Cedex, France \\ ${ }^{5}$ UK Astronomy Technology Centre, Royal Observatory, Blackford Hill, Edinburgh EH9 3HJ, UK \\ ${ }^{6}$ Institute for Astronomy, University of Edinburgh, Blackford Hill, Edinburgh EH9 3HJ, UK \\ ${ }^{7}$ Cavendish Laboratory, University of Cambridge, 19 J.J. Thomson Avenue, Cambridge CB3 OHE, UK \\ ${ }^{8}$ Kavli Institute for Cosmology, University of Cambridge, Madingley Road, Cambridge CB3 OHA, UK \\ ${ }^{9}$ Astronomy Department, California Institute of Technology, MC 249-17, 1200 East California Boulevard, Pasadena, CA 91125, USA \\ ${ }^{10}$ Department of Astronomy, Cornell University, Ithaca, NY 14853, USA \\ ${ }^{11}$ Institute for Computational Cosmology, Durham University, South Road, Durham DH1 3LE, UK \\ ${ }^{12}$ Max-Planck-Institut für Radioastronomie, Auf dem Hügel 69, D-53121 Bonn, Germany \\ Received 2012 June 26; accepted 2013 January 2; published 2013 February 11
}

\begin{abstract}
Modern (sub-)millimeter/radio interferometers such as ALMA, JVLA, and the PdBI successor NOEMA will enable us to measure the dust and molecular gas emission from galaxies that have luminosities lower than the Milky Way, out to high redshifts and with unprecedented spatial resolution and sensitivity. This will provide new constraints on the star formation properties and gas reservoir in galaxies throughout cosmic times through dedicated deep field campaigns targeting the $\mathrm{CO} /[\mathrm{C}$ II] lines and dust continuum emission in the (sub-)millimeter regime. In this paper, we present empirical predictions for such line and continuum deep fields. We base these predictions on the deepest available optical/near-infrared Advanced Camera for Surveys and NICMOS data on the Hubble Ultra Deep Field (over an area of about $12 \operatorname{arcmin}^{2}$ ). Using a physically motivated spectral energy distribution model, we fit the observed optical/near-infrared emission of 13,099 galaxies with redshifts up to $z=5$, and obtain median-likelihood estimates of their stellar mass, star formation rate, dust attenuation, and dust luminosity. We combine the attenuated stellar spectra with a library of infrared emission models spanning a wide range of dust temperatures to derive statistical constraints on the dust emission in the infrared and (sub-)millimeter which are consistent with the observed optical/near-infrared emission in terms of energy balance. This allows us to estimate, for each galaxy, the (sub-)millimeter continuum flux densities in several ALMA, PdBI/NOEMA, and JVLA bands. As a consistency check, we verify that the $850 \mu \mathrm{m}$ number counts and extragalactic background light derived using our predictions are consistent with previous observations. Using empirical relations between the observed $\mathrm{CO} /[\mathrm{CII}]$ line luminosities and the infrared luminosity of star-forming galaxies, we infer the luminosity of the $\mathrm{CO}(1-0)$ and $\left[\mathrm{C}_{\mathrm{II}}\right]$ lines from the estimated infrared luminosity of each galaxy in our sample. We then predict the luminosities of higher $\mathrm{CO}$ transition lines $\mathrm{CO}(2-1)$ to $\mathrm{CO}(7-6)$ based on two extreme gas excitation scenarios: quiescent (Milky Way) and starburst (M82). We use our predictions to discuss possible deep field strategies with ALMA. The predictions presented in this study will serve as a direct benchmark for future deep field campaigns in the (sub-)millimeter regime.
\end{abstract}

Key words: dust, extinction - galaxies: evolution - galaxies: ISM - galaxies: statistics - submillimeter: galaxies

Online-only material: color figures, machine-readable tables

\section{INTRODUCTION}

The last decade has seen impressive advances in our understanding of galaxy formation and evolution through galaxy surveys done (preferentially) at optical and infrared wavelengths. In particular, the history of star formation (the "Lilly-Madau" plot; e.g., Lilly et al. 1996; Madau et al. 1996; Hopkins \& Beacom 2006), and the buildup of stellar mass as a function of galaxy type and mass, have been well quantified to within $1 \mathrm{Gyr}$ of the Big Bang. It has been shown that the comoving cosmic star formation rate (SFR) density likely increases gradually from $z \sim 6$ to 10 , it peaks at $z \simeq 2$, and drops by more than an order of magnitude from $z \simeq 1$ to $z \simeq 0$ (Hopkins \& Beacom 2006; Bouwens et al. 2010). The buildup of stellar mass follows this evolution, as does the temporal integral (Bell et al. 2003; Ilbert et al. 2010). The redshift range $z \simeq 1-3$, during which roughly half of the stars in the Universe were formed, is referred to as the "epoch of galaxy assembly." In summary, the star formation properties as well as the stellar masses of galaxies have been well delineated through these optical and near-infrared deep field studies; almost all of our current knowledge is based on optical and near-infrared deep fields of the stars, star formation, and ionized gas (but, see, e.g., Smolčić et al. 2009; Karim et al. 2011 for additional constraints based on radio continuum studies). For example, Lyman Break Galaxy samples have revealed a major population of star-forming galaxies at $z \simeq 3$ (e.g., Steidel et al. 2003). Likewise, magnitude-selected samples (e.g., Le Fèvre et al. 2005; Lilly et al. 2007) provide a census of the star-forming population based on UV/optical flux rather than color. 
A key measurement that is currently (mostly) unavailable is that of the presence of molecular gas, i.e., the dense interstellar medium (ISM) phase ("fuel") required for star formation to take place, which lies at the heart of the evolution of the comoving cosmic SFR density. In recent years, there have been significant efforts devoted to obtaining molecular gas measurements of individual galaxies, by performing follow-up studies of galaxies that have been pre-selected from optical/NIR deep surveys. To date, color-selection techniques (e.g., "BzK," "BMBX") have revealed significant samples of gas-rich, star-forming galaxies at $z \simeq 1.5-2.5$ (with molecular gas masses $M_{\mathrm{H}_{2}} \simeq 10^{10}-10^{11} M_{\odot}$, stellar masses $M_{*} \simeq 10^{10}-10^{11} M_{\odot}$, and star formation rates SFR $\simeq 100 M_{\odot} \mathrm{yr}^{-1}$; Daddi et al. 2008, 2010a, 2010b; Genzel et al. 2010; Tacconi et al. 2010; Geach et al. 2011). While very important in their own right, these studies (which focus on the detection of carbon monoxide, the main tracer for molecular gas at low and high redshift) remain fundamentally limited to galaxy populations that were pre-selected in the optical/near-infrared, i.e., potentially missing gas-dominated and/or obscured systems.

From a theoretical/modeling perspective, Obreschkow et al. (2009a, 2009b, 2011) provide simulations of the cosmic evolution of the molecular (and atomic) hydrogen in galaxies as a function of redshift, by building on the Millennium dark matter simulations (Springel et al. 2005) in which they place "idealized model galaxies" at the centers of the dark matter halos which then evolve according to simple rules ("semi-analytical modeling;" Obreschkow et al. 2009a, 2009b, 2011). Power et al. (2010) and Lagos et al. (2011) also present models of the cosmic evolution of the atomic and molecular gas content in galaxies by applying different semi-analytical galaxy formation models to the Millennium simulation.

In this paper, we present empirical predictions of molecular line and continuum deep fields that are only now becoming possible thanks to the advent of observational facilities that dwarf previous capabilities, in particular the broad bandwidth and sensitivity afforded by the Atacama Large Millimeter Array (ALMA), the Jansky Very Large Array (JVLA; formerly known as EVLA), and the IRAM Plateau de Bure Interferometer (PdBI) successor, the Northern Extended Millimeter Array (NOEMA). Our predictions are based on the deepest available optical and near-infrared data available for the Hubble Ultra Deep Field (UDF), but, barring cosmic variance, should give a statistical representation of an arbitrarily chosen region on the sky. Basically, we use a sophisticated spectral energy distribution (SED) model combined with a Bayesian approach (da Cunha et al. 2008) to interpret the observed optical/nearinfrared emission of the UDF galaxies in terms of their stellar content, star formation activity, and dust attenuation, and obtain statistical constraints on their total dust luminosity which are consistent with the observed stellar emission in terms of energy balance (i.e., all the stellar radiation absorbed by dust in the rest-frame ultraviolet to near-infrared is re-emitted in the midinfrared to millimeter range). The dust luminosity of each model is then re-distributed at infrared to millimeter wavelengths by combining the (dust-attenuated) stellar SED with a library of infrared dust emission models spanning a wide range of dust SED shapes (including different dust temperatures). This allows us to derive median-likelihood estimates of the (sub-)millimeter continuum flux densities of our galaxies in several ALMA, PdBI/NOEMA, and JVLA bands. Based on these continuum predictions, we calculate predicted line strengths in the various rotational transitions of carbon monoxide ( $\mathrm{CO})$ and ionized carbon ([C II]), two main tracers of the star-forming ISM in galaxies. We note that, with this technique, we potentially miss, by definition, extremely dust obscured sources that are not included in our optical/near-infrared catalog. However, this should not have a great impact in our results since the main goal of this paper is to characterize the general galaxy population rather than the extreme, dust-enshrouded starbursts.

In Section 2, we describe the main optical/near-infrared photometric catalog of the Hubble UDF in which we base our predictions, as well as additional data from optical, infrared, and submillimeter surveys of the UDF/Extended Chandra Deep Field South (ECDF-S) area that we use to test our predictions. In Section 3, we describe our spectral energy model and fitting method. In Section 4, we analyze in detail our predicted (sub-)millimeter properties for 13,099 galaxies in the UDF. We discuss the SED fitting outputs and derived physical properties of the galaxies in our sample in Sections 4.1 and 4.2, respectively. In Section 4.3, we discuss the reliability of our infrared luminosity and (sub-)millimeter continuum flux estimates from the observed optical/near-infrared SEDs, and in Sections 4.4 and 4.5 we perform consistency checks on the predicted continuum flux densities of our galaxies at $850 \mu \mathrm{m}$ by comparing them with observed number counts and the extragalactic background light (EBL). In Section 4.6, we present the distribution of our predicted continuum flux densities for the whole sample in several (sub-)millimeter bands from 38 to $870 \mathrm{GHz}$, and in Section 4.7 we infer the $\mathrm{CO}$ and [C II] line luminosities of the galaxies in our sample from the estimates of their infrared luminosities, based on simple empirical relations. Based on these (sub-)millimeter line and continuum predictions, we discuss future deep fields with ALMA in Section 5. We summarize our main conclusions in Section 6. Appendix A contains a comparison of our results with what we would obtain assuming fixed SED shapes for the galaxies in our sample.

Throughout this paper, we use a concordance $\Lambda$ CDM cosmology with $H_{0}=70 \mathrm{~km} \mathrm{~s}^{-1} \mathrm{Mpc}^{-1}, \Omega_{\Lambda}=0.7$, and $\Omega_{m}=0.3$.

\section{THE DATA}

As an example, we here base our predictions on the deepest available optical and infrared data on the Hubble UDF that will be accessible with ALMA. We stress that this coherent database is the only reason for our choice and that, barring the issue of cosmic variance, we could have used any other field for our predictions as well. This means that our statistical predictions should also hold for a northern field that will be accessible from other telescopes (e.g., IRAM PdBI/NOEMA, JVLA).

\subsection{Input Catalog: Optical/Near-infrared HST Data}

We start by using the photometric catalog of the Hubble UDF (centered at R.A. $=03^{\mathrm{h}} 32^{\mathrm{m}} 39^{\mathrm{s}} .0$, decl. $\left.=-27^{\circ} 47^{\prime} 29^{\prime \prime} .1\right)$ described in Coe et al. (2006). This contains aperture-matched, point-spread-function (PSF)-corrected Advanced Camera for Surveys (ACS) $B V i^{\prime} z^{\prime}$ and NICMOS3 $J H$ photometry, as well as Bayesian photometric redshifts for all the detected sources, accurate to within $0.04\left(1+z_{\text {spec }}\right)$ (Coe et al. 2006). The full catalog contains 18,700 sources, of which a large fraction (8042) are detected at the $10 \sigma$ level in at least one band. The $10 \sigma$ limiting $\mathrm{AB}$ magnitudes in the $B, V$, $i^{\prime}, z^{\prime}, J$, and $H$ are 28.71, 29.13, 29.01, 28.43, 28.30, and 28.22, respectively. Following Coe et al. (2006), to exclude contamination from stars, we exclude sources with $i$-band stellarity stel $\geqslant 0.8$ (about $6 \%$ of the sample), leaving us 

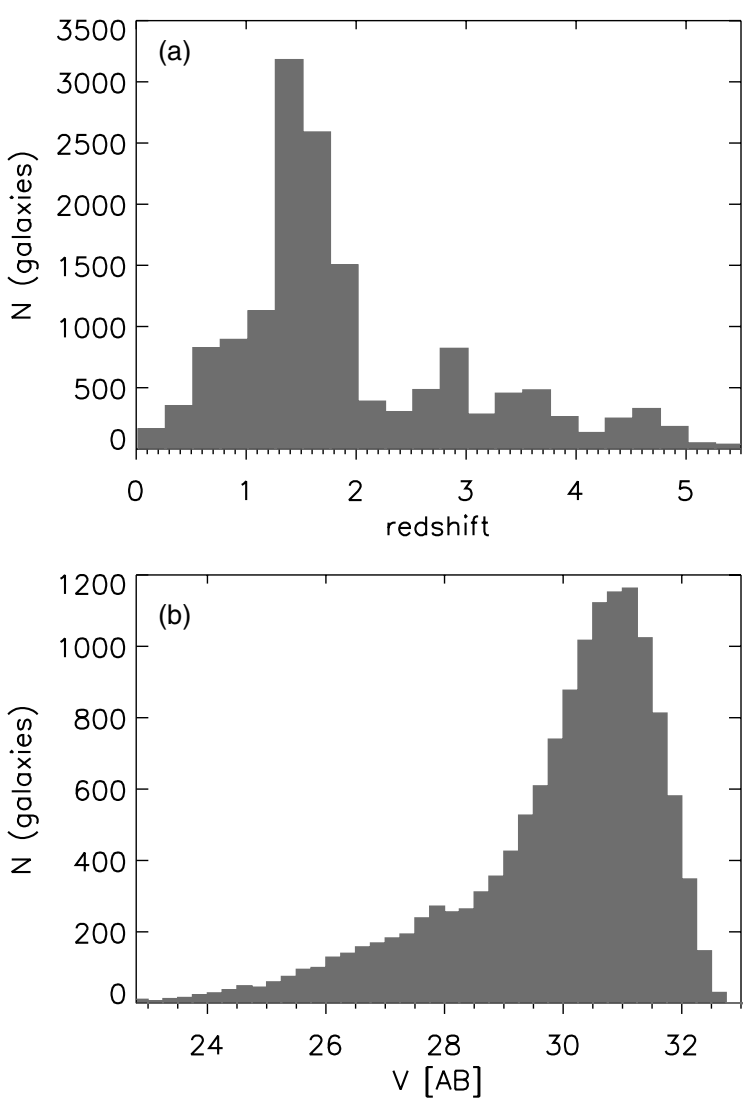

Figure 1. Distribution of the photometric redshift (a) and $V$-band magnitude (b) of the optically selected Hubble UDF galaxies from the Coe et al. (2006) catalog.

with 17,532 galaxies, with photometric redshifts distributed as plotted in Figure 1(a). For reference, in Figure 1(b), we plot the distribution of the observed ACS $V$-band magnitude for 16,830 sources detected in that band. We note that the fact that the redshift distribution of our sources peaks at $z \simeq 2$ and the sudden drop in sources fainter than $30 \mathrm{AB}$ magnitudes in the $V$ band are due to incompleteness. This implies that our predictions may be missing high-redshift, dust-obscured galaxies that are too faint in the optical to be in our sample and may have large (sub-)millimeter fluxes. This is the case of LESSJ0333243.6274644 , the only submillimeter source detected in the UDF as part of the LESS survey (LABOCA observations of the ECDF-S at $870 \mu \mathrm{m}$; Weiß et al. 2009), which has no optical counterpart in our optical catalog. Our predictions are therefore lower limits for the possible number of detections at high redshift $(z>2)$.

\subsection{Supporting Data}

We complement the photometry in the UDF catalog with additional photometry out to the far-infrared. We use 54 galaxies detected in the Herschel/SPIRE bands available in the publicly released HerMES survey (PI: S. Oliver; Oliver et al. 2010) images in GOODS-South, for which we applied the same prior source extraction technique as in Elbaz et al. (2011) for the GOODS-Herschel SPIRE data in GOODS-North. Herschel/PACS images of the GOODS-South are available as part of the GOODS-Herschel program (PI: D. Elbaz). For each of these $250 \mu \mathrm{m}$ selected galaxies, well-sampled SEDs from the ultraviolet to the far-infrared are available, including photometry in the following bands: $U, B, V, i, z, J, K$, Spitzer/IRAC 3.6, 4.5, 5.8, and $8.0 \mu \mathrm{m}$, Spitzer/IRS at $16 \mu \mathrm{m}$, Spitzer/MIPS at $24 \mu \mathrm{m}$, Herschel/PACS at 70, 100, and $160 \mu \mathrm{m}$, and
Herschel/SPIRE at 250, 350, and $500 \mu \mathrm{m}$ (Elbaz et al. 2011; Magdis et al. 2011). The redshifts of the galaxies in this subsample go from $z=0.140$ to $z=2.578$, with a median of value of 1.0. We use this sub-sample in Section 4.3 to test the reliability of our predictions of the total infrared luminosity and (sub-)millimeter continuum fluxes from observed optical data as described in Section 3.

To test our predictions for a wider field and address the issue of cosmic variance in Section 4.4, we use a wider-area catalog of the CDF-S field which also includes the UDF but is about 10 times larger in area, the FIREWORKS catalog (Wuyts et al. 2008). The FIREWORKS catalog is a $K_{s}$-band-selected galaxy catalog which contains multi-wavelength photometry of 6,308 galaxies from the $U$ band to the Spitzer $24 \mu \mathrm{m}$ band, with a $5 \sigma$ magnitude limit of $24.3 \mathrm{AB}$ mag in the $K_{s}$ band (i.e., shallower than the UDF Hubble Space Telescope (HST) catalog described in Section 2.1), over $138 \operatorname{arcmin}^{2}$.

\section{SED MODELING}

In a next step we use the models described in da Cunha et al. (2008) to fit the observed rest-frame optical to near-infrared SEDs of the galaxies from the photometric catalog described in Section 2.1, and obtain statistical estimates of the infrared luminosities, (sub-)millimeter continuum flux densities, and CO line luminosities for each galaxy in the sample.

\subsection{Ultraviolet to Near-infrared Emission}

We use the spectral synthesis model of Bruzual \& Charlot (2003) to compute the integrated light emitted by stars in galaxies for a wide range of metallicities (distributed between 0.02 and 2 times solar), ages (distributed between $0.1 \mathrm{Gyr}$ and the age of the Universe at each redshift), and star formation histories (parameterized as exponentially declining with a wide range of timescales, and superimposed random bursts of star formation). To account for the attenuation of starlight by dust, we describe the ISM of galaxies using the two-component model of Charlot \& Fall (2000): the ambient (diffuse) ISM and the starforming regions (birth clouds). This prescription accounts for the fact that stars are born in dense molecular clouds, which dissipate typically on a timescale of $10^{7} \mathrm{yr}$. As a result, the non-ionizing continuum emission from young $\mathrm{OB}$ stars and line emission from their surrounding $\mathrm{H}$ iI regions is absorbed by dust in these birth clouds and then in the ambient ISM, while the light emitted by stars older than $10^{7} \mathrm{yr}$ propagates only through the diffuse ISM. The main free parameters of this model are the effective $V$-band optical depth seen by young stars in birth clouds, $\hat{\tau}_{V}$, and the fraction of $\hat{\tau}_{V}$ contributed by dust in the diffuse ISM, $\mu$. Using this model, we compute the attenuated stellar emission of galaxies and the total luminosity absorbed and re-radiated by dust in the birth clouds and the diffuse ISM. We use the model library described in da Cunha et al. (2008), which includes 50,000 attenuated stellar spectra spanning a wide range of star formation histories, metallicities, and dust optical depths.

\subsection{Infrared to Millimeter Emission}

In the context of the model described in da Cunha et al. (2008), the energy absorbed by dust is re-radiated at infrared wavelengths through four different components:

1. the emission by polycyclic aromatic hydrocarbons (PAHs),

2. a hot mid-infrared continuum (with temperature in the range $130-250 \mathrm{~K}$ ), 
3. emission by warm dust in thermal equilibrium (with temperature in the range $30-60 \mathrm{~K}$ and dust emissivity index $\beta=1.5$ ),

4. emission by cold dust in thermal equilibrium (with temperature in the range $15-25 \mathrm{~K}$ and dust emissivity index $\beta=2$ ).

da Cunha et al. (2008) use a wide library of infrared emission spectra where the temperatures and relative contributions of each component to the total infrared luminosity span a wide range of values. The models in this library are then directly compared to infrared observations of galaxies to constrain each dust emission component. In this paper, since we hardly have any observational constraints on the infrared SED of the galaxies, we do not require such a wide range of models. Therefore, we will adopt a reduced set of dust emission models that reflect the range of infrared SED shapes of local, normal star-forming galaxies.

For this work, our goal is to obtain a range of possible infrared SEDs that are consistent with the observed optical/near-infrared emission in terms of their overall energy balance. Therefore, for simplicity, we fix the values of most free parameters controlling the shape of the infrared SEDs of galaxies to those of three representative infrared SEDs presented in da Cunha et al. (2008): a "standard" model (with equilibrium temperatures of the cold and warm dust components 22 and $48 \mathrm{~K}$, respectively), a "hot" model (25 and $55 \mathrm{~K})$ and a "cold" model (18 and $40 \mathrm{~K})$; the relative contributions of the cold and warm dust components, as well as the PAHs and hot mid-infrared continuum are different for each model and are listed in Table 1 of da Cunha et al. (2008). These three models were calibrated using observed IRAS and $I S O$ infrared fluxes of local star-forming galaxies and span the range of observed infrared colors for this low-redshift sample. The "standard" model reproduces the median infrared colors of local galaxies, the "hot" model is representative of the warmest observed infrared colors, and the "cold" model is representative of the cooler infrared colors. We build a simplified dust emission model library in which we fix the values of the dust temperatures and the contribution by PAHs, hot mid-infrared continuum and warm dust to the total luminosity of birth clouds, as well as the contribution of cold dust to the total dust luminosity of the diffuse ISM, to the values of these representative models, while leaving the fraction of total dust luminosity contributed by the diffuse ISM, $f_{\mu}=L_{\text {dust }}^{\text {ISM }} / L_{\text {dust }}$, and the total dust luminosity, $L_{\text {dust }}$ as free parameters.

\subsection{Radio Continuum}

In addition to thermal dust emission, the (sub-)millimeter continuum emission of star-forming galaxies can have a nonnegligible radio continuum emission component, especially at the lowest frequencies. This emission is mainly free-free emission from $\mathrm{H}$ II regions and synchrotron radiation from relativistic electrons accelerated in supernova remnants (e.g., Condon 1992). Since the da Cunha et al. (2008) models do not include radio emission, we add a radio continuum component to our model SEDs in order to account for this extra contribution to the (sub-)millimeter continuum. We use the simple prescription described in Dale \& Helou (2002), which is based on the observed radio/far-infrared correlation. The radio/far-infrared correlation (e.g., Helou et al. 1985; Condon 1992; Bell 2003) implies that the ratio between the observed far-infrared flux of a galaxy (between $42.5 \mu \mathrm{m}$ and $122.5 \mu \mathrm{m}$ ) and the radio flux density at $1.4 \mathrm{GHz}, q$, is constant. We model the radio continuum in star-forming galaxies as a sum of two main components:

1. a thermal component, consisting mainly of free-free emission from ionized gas, with spectral shape $f_{v}^{\text {th }} \propto v^{-0.1}$;

2. a non-thermal component, consisting of synchrotron radiation, with spectral shape $f_{v}^{\text {nth }} \propto v^{-0.8}$.

In normal star-forming galaxies, the contribution of the thermal (free-free) component to the radio continuum at $20 \mathrm{~cm}$ is $\sim 10 \%$ (Condon 1992). This allows us to fix the spectral shape of our radio continuum, which we normalize relative to the farinfrared flux of each model in our library using the value found by Yun et al. (2001), $q=2.34$. This prescription is based on the assumption that the galaxies fall in the observed radio/farinfrared correlation, and has the advantage of not requiring any extra free parameters to estimate the radio continuum. We also assume that the radio/far-infrared correlation remains constant with redshift (e.g., Sargent et al. 2010).

\subsection{SED Fitting Method}

The libraries of attenuated stellar emission and dust emission are combined by associating models with similar values of $f_{\mu}=$ $L_{\text {dust }}^{\text {ISM }} / L_{\text {dust }}$ (the fraction of total dust luminosity contributed by the diffuse ISM), which are scaled to the same total dust luminosity $L_{\text {dust }}$. This ensures, for each model, the energy balance between the radiation absorbed and re-emitted by dust in the diffuse ISM and stellar birth clouds.

For each galaxy, we compare the observed optical fluxes in the ACS and NICMOS bands (Section 2) to the predicted fluxes for every model of the stochastic library described above, by computing the $\chi^{2}$ goodness of fit for each model. We then build the likelihood distribution of any given physical parameter for the observed galaxy by weighting the value of that parameter in each model by the probability $\exp \left(-\chi^{2} / 2\right)$. Our final estimate of the parameter is the median of the likelihood distribution, with an associated confidence interval which is the 16th-84th percentile range of the distribution (this confidence interval is tighter for well-constrained parameters and wider when the parameters are not well constrained by the available observations). In what follows, the values of the physical properties of the galaxies mentioned refer to the median values of the probability density distribution. We also obtained the best-fit model SED for each galaxy, which is the model that minimizes $\chi^{2}$.

\section{PREDICTED (SUB-)MILLIMETER PROPERTIES}

\subsection{SED Fitting Outputs}

We use the method described above to fit the observed photometry of each galaxy and produce likelihood distributions of their stellar mass, SFR, dust optical depths, infrared luminosities, dust masses, continuum, and molecular line fluxes in the (sub-)millimeter range.

In Figure 2, we show an example SED fit and the associated likelihood distributions of some physical parameters: the SFR, stellar mass $\left(M_{*}\right)$, dust luminosity $\left(L_{\text {dust }}\right)$, dust mass $\left(M_{\text {dust }}\right)$, far-infrared luminosity ( $L_{\mathrm{FIR}}$, defined as the integral of the infrared emission between 42.5 and $122.5 \mu \mathrm{m}$ ), and the continuum flux densities in a number of accessible (sub-)millimeter windows at 345, 230, and $100 \mathrm{GHz}$ (specifically, ALMA bands 7, 6 , and 3, respectively, and PdBI/NOEMA band 1, 3, and 4). The ultraviolet to near-infrared part of the SED is the stellar population model that best fits the data. The far-infrared and 

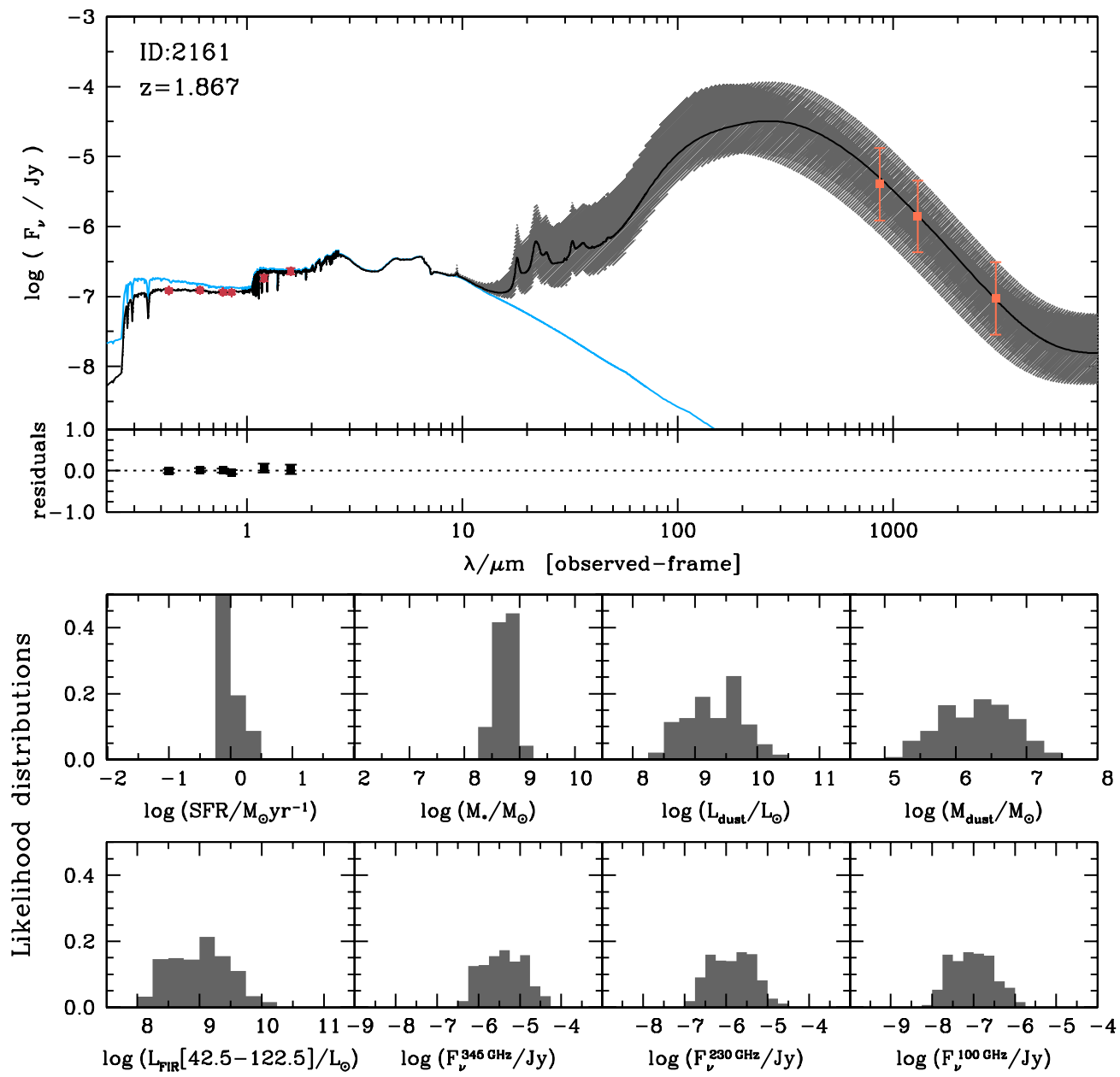

Figure 2. Example of a fit to the observed optical spectral energy distribution of a UDF galaxy at $z=1.867$ (red points). The black line represents the best-fit model SED; the blue line represents the unattenuated (i.e., dust-free) stellar spectrum. The gray-shaded area represents the range of all infrared dust emission models in our model library that are consistent with the observed optical/near-infrared fluxes in terms of energy balance. The orange points represent the median of the probability density function (PDF) for the predicted fluxes at 345, 230, and $100 \mathrm{GHz}$ (three arbitrary chosen (sub-)millimeter bands), and the associated error bars represent the confidence range, i.e., the 16th-84th percentile range of the PDF. The residuals of the fit are plotted in the panel at the bottom of the SED. The eight bottom panels show the PDFs of several parameters: star formation rate; stellar mass; total dust luminosity; dust mass; far-infrared luminosity between 42.5 and $122.5 \mu$ m; and flux densities in the three randomly chosen bands at 345,230 , and $100 \mathrm{GHz}$.

(A color version of this figure is available in the online journal.)

(sub-)millimeter part of the SED that is plotted corresponds to the model with the best-fit $L_{\text {dust }}$ and $f_{\mu}$, but all the other parameters controlling the shape of the SED at these wavelengths are randomly drawn from the library of dust emission models. The gray-shaded area shows the range of possible infrared SEDs allowed within the uncertainties in dust luminosity with different dust temperatures and contributions of PAHs, mid-IR continuum and dust in thermal equilibrium reflecting the diversity of possible dust emission models in our model library. The orange points show the (exemplary) median-likelihood estimates of the flux densities at 345,230 , and $100 \mathrm{GHz}$, where the error bars (16th-84th percentile range) reflect all the different combinations of infrared models that are consistent with the observed optical data.

We impose a minimum of three photometric bands to define the SED, and we discard galaxies with $z>5$ and galaxies for which the fit residuals are larger than $2 \sigma$ (where $\sigma$ is the uncertainty associated with the flux measurement) in each band. These criteria allow us to discard the most unreliable SED fits: at very high redshift, our model becomes uncertain and the observations sample only the far-UV emission, making it very difficult to constrain the SED; also, galaxies with very high residuals in a given band may indicate problems with the photometry or wrong photometric redshift, or the presence of an active galactic nucleus (AGN, which is not included in our models). Our final sample used in the remainder of this paper consists of 13,099 sources with $z \leqslant 5$.

\subsection{Derived Physical Properties}

Stellar masses are constrained to within \pm 0.35 dex, which reflects uncertainties due to the fact that, for most of our galaxies, observations do not include the rest-frame near-infrared, where the light is dominated by low-mass stars, which constitute the bulk of the stellar mass in galaxies. However, we show in the next section (Section 4.3) that this does not cause any systematic effects on the stellar mass estimates. The SFRs are constrained to within typically $\pm 0.2 \mathrm{dex}$, due to the fact that the observed SEDs sample the emission by young stars in the rest-frame ultraviolet. The dust luminosities are more uncertain (confidence ranges are typically $\pm 0.45 \mathrm{dex}$ ), as expected due to the lack of infrared observations for our sample. The dust 

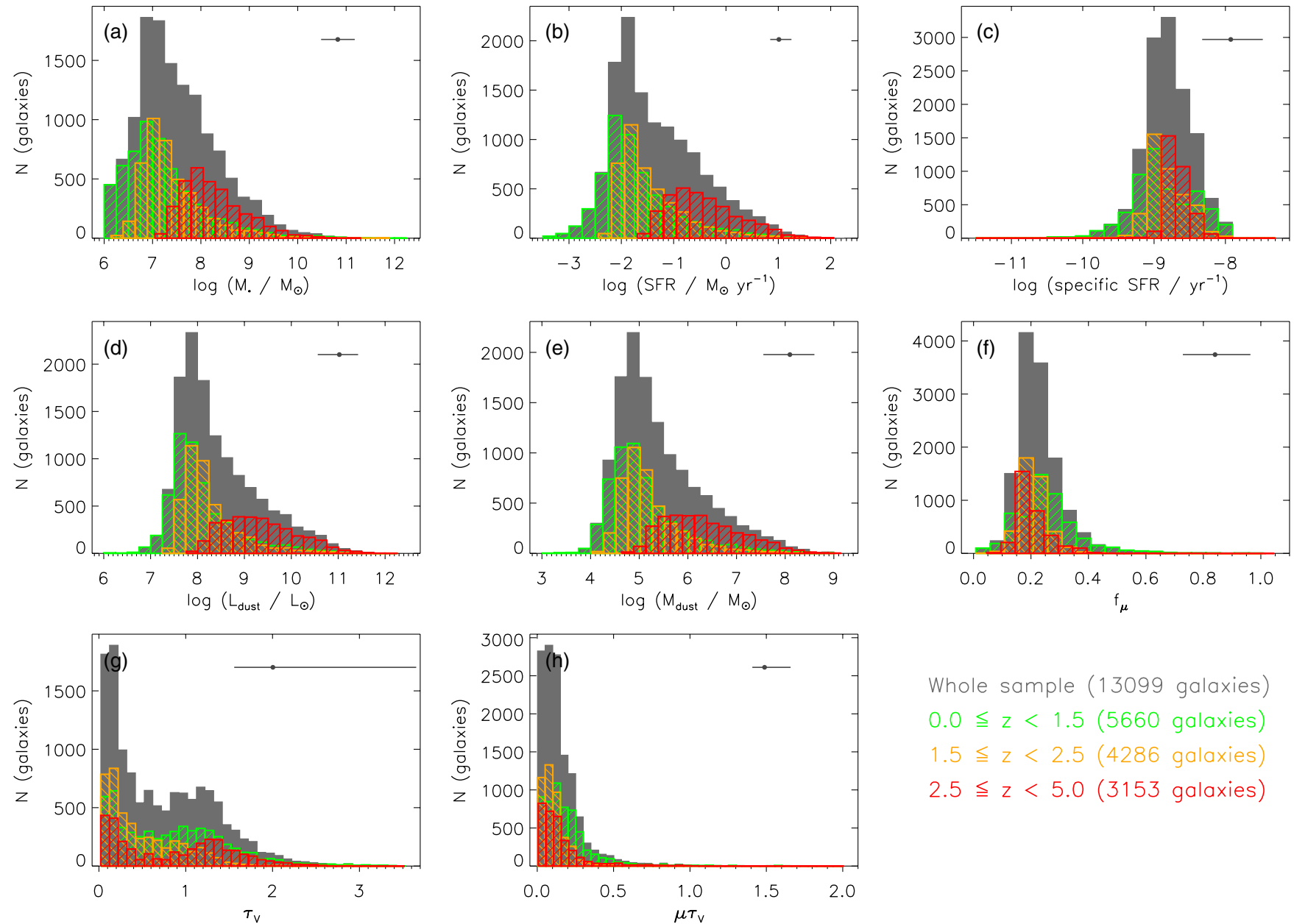

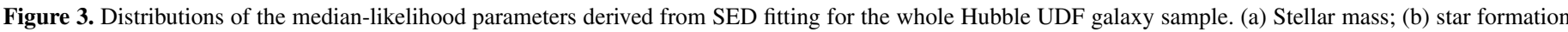

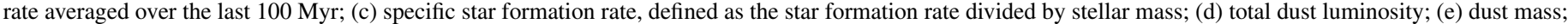

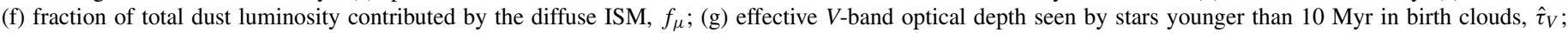

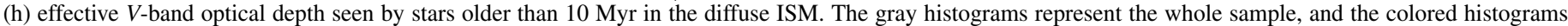

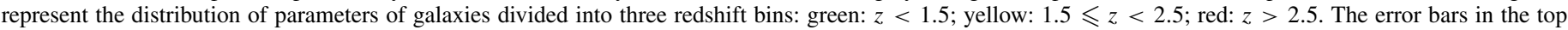

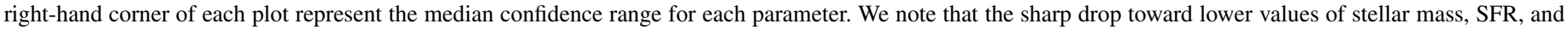
dust mass/luminosity is due to incompleteness of the photometric catalog toward fainter flux levels (see Figure 1).

(A color version of this figure is available in the online journal.)

luminosity is estimated by our model by calculating the total energy absorbed by dust, taking into account the light emitted by stars and the attenuation by dust. Therefore, by construction, our dust luminosities are consistent with all stellar and dust attenuation parameters (SFR, $M_{*}, \mu, \hat{\tau}_{V}, f_{\mu}$ ), from an energybalance perspective (as described in da Cunha et al. 2008 and in Section 3). Even though we have significant uncertainties in the dust luminosity estimates, as expected from our sparse SED sampling, we are still able to predict $L_{\text {dust }}$ well within an order of magnitude (we analyze possible systematic effects in Section 4.3). The dust masses are also estimated by using all the dust emission model templates that are consistent with the statistical estimates on dust luminosities. The confidence range for $M_{\text {dust }}$ is very large $( \pm 0.55 \mathrm{dex})$, and reflects not only the uncertainty in $L_{\text {dust }}$, but also the large uncertainty in infrared SED shapes and dust temperatures. These dust masses are merely indicative of the range of dust masses that are consistent with the observed SEDs in terms of energy balance, and taking into account a range of possible dust temperatures, $18-25 \mathrm{~K}$ for the cold dust (with $\beta=2$ ), and 40-55 K for the warm dust (with $\beta=1.5$ ).
The distributions of physical parameters inferred from our SED fits (Figure 3) show that the bulk of our galaxies are low-mass, low SFR, and low dust attenuation $\left(\hat{\tau}_{V}\right.$ and $\left.\mu \hat{\tau}_{V}\right)$ sources, i.e., blue star-forming galaxies (consistent with the finding of a large population of faint blue galaxies in the UDF described in Coe et al. 2006). As expected, galaxies in the highest redshift bin, $z \geqslant 2.5$, have typically higher stellar masses and SFRs, because only the brightest galaxies are detected. The median dust luminosity of the sources, $L_{\text {dust }}$ increases from $\log \left(L_{\text {dust }} / L_{\odot}\right) \simeq 8.0$ in the lowest redshift bins to $\log \left(L_{\text {dust }} / L_{\odot}\right) \simeq 9.2$ at $z \geqslant 2.5$. Figures $3(\mathrm{~g})$ and (h) show that this is not necessarily due to an increase in the dust optical depth of galaxies in the highest redshift bin, but rather to the fact that the galaxies detected have larger stellar masses and SFRs, as shown in Figures 3(a) and (b).

\subsection{Reliability of Infrared Luminosity Estimates}

It is important to test how well we can predict the total infrared luminosity of the galaxies in our sample from their observed restframe UV/optical SEDs. To do so, we use the sample of 54 UDF 

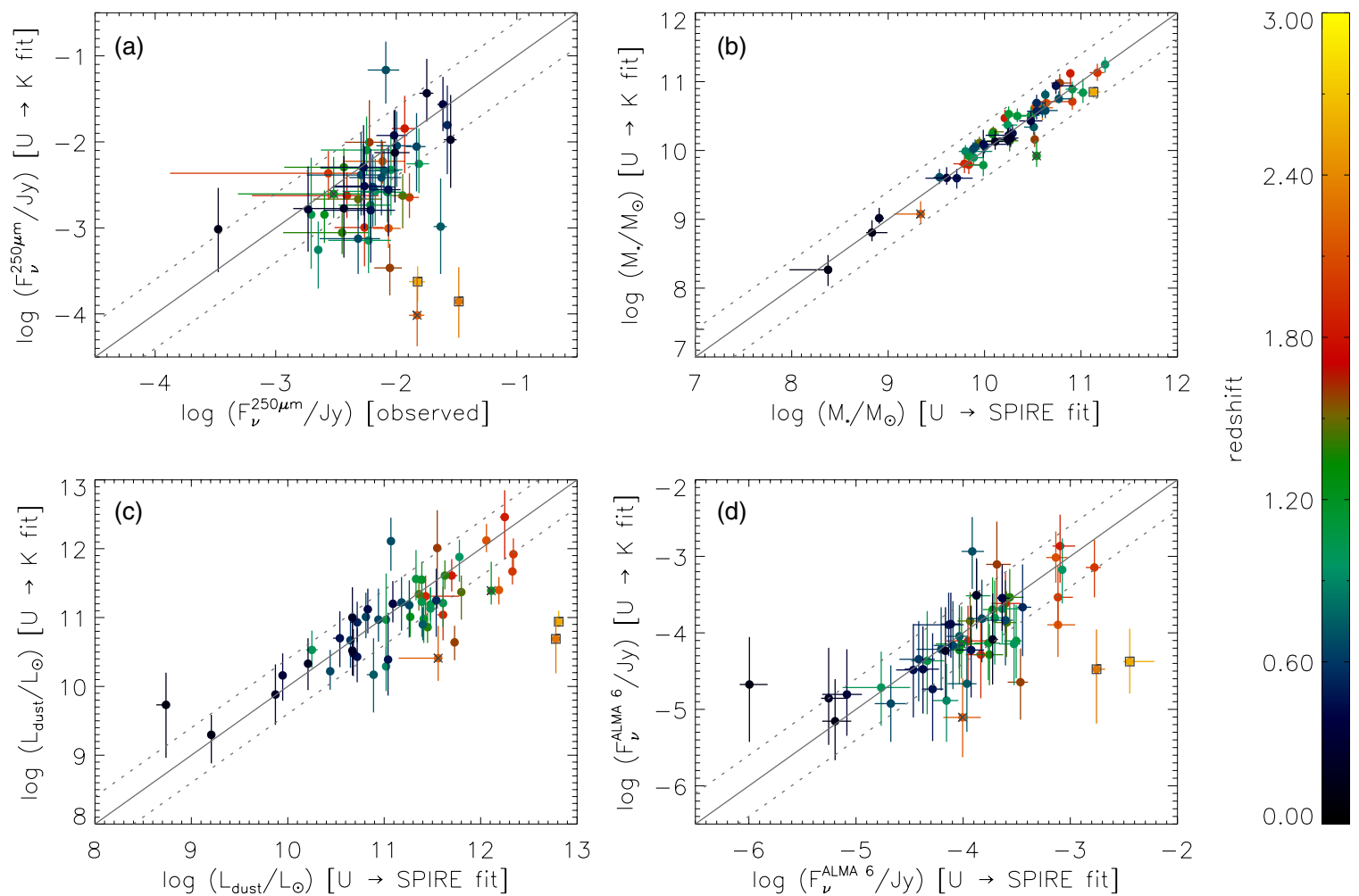

Figure 4. (a) Comparison between the observed Herschel/SPIRE $250 \mu \mathrm{m}$ flux ( $x$-axis) and our Bayesian median-likelihood estimate of the $250 \mu \mathrm{m}$ flux of each galaxy based on SED fits from the $U$ band to the $K$ band ( $y$-axis). The other three panels show the comparison between our Bayesian median-likelihood estimates of GOODS/Herschel galaxy parameters obtained when fitting the full SED from the $U$ band to the longest available SPIRE band ( $x$-axis), and when fitting only the SED from the $U$ band to the $K$ band ( $y$-axis): (b) stellar mass; (c) total dust luminosity; (d) continuum flux in the ALMA band 6 (230 GHz). Each galaxy is color-coded according to redshift. The error bars show the 16th-84th percentile range of the likelihood distributions. In all panels, the gray solid line is the identity line, and the dotted lines show a \pm 0.5 dex offset for reference. The two points marked with crosses are galaxies that show a significant AGN contribution in the infrared; the two points marked with squares are galaxies which show a ULIRG-like SED, i.e., they seem to be very optically thick (given their high intrinsic infrared-to-optical emission ratios). Our SED modeling may not be reliable for these four galaxies, but overall we find a good agreement between the estimates derived from fitting the full SED and those from fitting the SED only up to the $K$ band.

(A color version of this figure is available in the online journal.)

galaxies detected in the Herschel/PACS and Herschel/SPIRE bands as part of the GOODS-Herschel program described in Section 2.2. For each of these galaxies, we have well-sampled SEDs from the ultraviolet to the far-infrared, which allows us to test our SED extrapolations. We fit the observed (more complete) SEDs of this sub-sample of galaxies using the same method as described in Section 3.4. First, we include the full observed ultraviolet to far-infrared observations in the SED fits, not only to check that our model can reproduce consistently the SEDs of these galaxies, but also to get the best possible estimates of the stellar masses, SFRs, dust luminosities, and continuum (sub-)millimeter fluxes for these sources. Then, we re-fit the SEDs using only observations between the $U$ band and the $K$ band, to mimic the set of observations available for the majority of galaxies discussed above.

In Figure 4(a), we compare the median likelihood of the Herschel/SPIRE $250 \mu \mathrm{m}$ flux derived from the fits from the $U$ to $K$ band with the actual observed $250 \mu \mathrm{m}$ flux for each galaxy. We find a small systematic offset of 0.25 dex between the observations and our estimates, in the sense that we tend to underestimate the $250 \mu \mathrm{m}$ flux of the galaxies on average with our $U$-band to $K$-band fits. However, for most of the galaxies, the observed value is still within the confidence range derived from our fits. This effect is likely to be less important for the total dust luminosity and (sub-)millimeter fluxes, as these do not depend as strongly on the exact location of the peak of the infrared SED (i.e., the actual dust temperature spanned by the models). In the next three panels of Figure 4, we compare the median likelihoods of the stellar masses, dust luminosities, and continuum ALMA band 6 fluxes obtained with the two sets of SED fits ( $U$-band to $K$-band fits versus $U$-to-SPIRE-fit). Figure 4(b) shows that the stellar masses agree remarkably well between the two fits (with a dispersion around the identity line of $0.13 \mathrm{dex}$ ). Not surprisingly, the total dust luminosities and predicted ALMA band 6 fluxes do not agree as well, as shown in Figures 4(c) and (d). The inclusion of infrared data in the fits helps constrain these properties better, as shown by the significantly reduced confidence ranges. In the case of $L_{\text {dust }}$, this happens because the infrared data allow us to constrain the bolometric dust luminosity by fitting the dust emission itself (as opposed to constraining $L_{\text {dust }}$ from the attenuated spectrum alone); the constraints on SFR are also tightened because we can account for dust-obscured SFR more accurately. The different far-infrared fluxes obtained with PACS and SPIRE help constrain the shape of the dust SED, namely, the dust temperatures and relative contributions of the warm and cold dust components to the total infrared emission. This helps obtaining tighter constraints on the (sub-)millimeter continuum fluxes (namely, the ALMA band 6 flux shown as an example in Figure 4(d)).

Even though the inclusion of infrared data helps constraining parameters such as the SFR, dust luminosity, and ALMA continuum fluxes, the median-likelihood estimates of these 
parameters when excluding the infrared data agree very well with the estimates derived from the full SED fits, even if, as expected, the associated confidence ranges are larger. We find very small offsets between the averages of the median-likelihood estimates derived from the two fits: 0.02 dex for SFR, 0.08 dex for $L_{\text {dust }}$, and 0.07 dex for the predicted ALMA band 6 continuum flux (in the sense of the $U$-band to $K$-band fits slightly underestimating the parameters), with a dispersion of $\simeq 0.40$ dex for all cases. These very small systematic offsets are well within our fit confidence ranges, and show that our approach to predict infrared luminosities and (sub-)millimeter continuum fluxes from modeling UV/optical SEDs is reliable. We note, however, that the difference between $250 \mu \mathrm{m}$ fluxes and total dust luminosities derived from the fits to the UV/optical data only and those measured with Herschel correlates with the dust optical depth in the galaxies. We tend to underestimate the (sub-)millimeter fluxes/dust luminosity when using only the $U$-band to $K$-band fits for galaxies with the highest dust attenuations (which translate into high infrared-to-optical ratios). This is due to the fact that our dust attenuation prior (Section 3; da Cunha et al. 2008) leads to an underestimation of the optical depth for extremely dust-enshrouded, starburst-like sources (such as local ultraluminous infrared galaxy (ULIRGs) or high-redshift submillimeter galaxies (SMGs); see da Cunha et al. 2010). While these galaxies can be a negligible fraction of our sample in number (e.g., Rodighiero et al. 2011; Sargent et al. 2012), they can dominate the bright (sub-)millimeter counts. This is the case for the two GOODS/Herschel sources in our sample with the highest redshift, which are marked in Figure 4 with squares. Due to the flux limit of this sample, at the highest redshifts $(z \simeq 2)$, only very dust-obscured ULIRGtype galaxies were selected. For this particular type of galaxies, the SED models used in Section 3 become limited. However, we expect this kind of galaxies to be rare in our optically selected sample of the UDF, and therefore we do not expect this limitation to greatly affect our results. We also note that our optically selected catalog is also likely to miss completely optically obscured galaxies (e.g., HDF850.1, Walter et al. 2012; GN10, Daddi et al. 2009a; GN20, Daddi et al. 2009b). The very good agreement between parameters derived from fits to the UV/ optical SED versus parameters derived from fits to the full SED is consistent with previous results showing that the star formation properties of normal star-forming galaxies up to $z \simeq 2$ can be reliably derived from UV/optical observations alone (e.g., Daddi et al. 2005, 2007; Reddy et al. 2006). This implies that the ISM of these galaxies is not heavily optically thick, and we can apply our energy-balance technique to interpret the SEDs of most normal star-forming "main-sequence" galaxies.

\subsection{Number Counts}

As a consistency check, we now compare our continuum flux density predictions with previously obtained number counts at (sub-)millimeter wavelengths.

Number counts at $850 \mu \mathrm{m}$ have been obtained using the SCUBA bolometer array on the James Clerk Maxwell Telescope and LABOCA on APEX by a number of groups over the last decade (e.g., Scott et al. 2002; Smail et al. 2002; Borys et al. 2003; Coppin et al. 2006; Scott et al. 2006; Knudsen et al. 2008; Weiß et al. 2009). These counts can be directly compared to our predictions at $345 \mathrm{GHz}$ (ALMA band 7, PdBI/NOEMA band 4) since this band probes roughly the same wavelength. In Figure 5, we compare our predicted cumulative number counts at $345 \mathrm{GHz}$

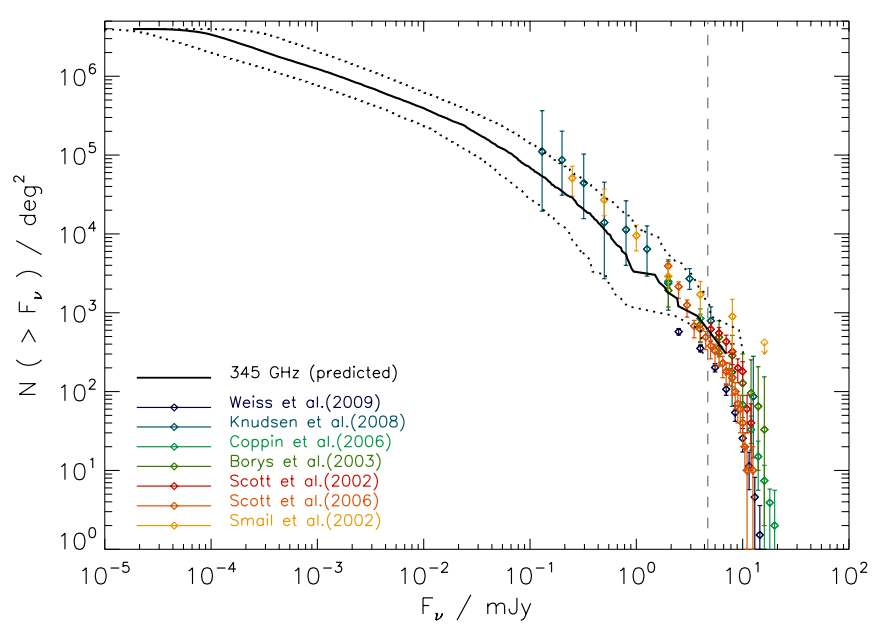

Figure 5. Predicted cumulative number counts in band 7 at $345 \mathrm{GHz}$ (black solid line), with confidence range estimated using the upper and lower flux limits given by the confidence range for each galaxy (dotted lines). The colored points show observed values at $\sim 850 \mu \mathrm{m}$ from different studies (see the figure legend for references). The vertical line shows the flux limit of the LESS survey catalog (Weiß et al. 2009), which includes the UDF.

(A color version of this figure is available in the online journal.)

with these previous observations. Our predicted number count range to the first order agrees with the observed number counts, but we do not reach the higher fluxes probed by submillimeter observations. The lack of the brightest sources is due to two reasons. First, the field on which we base our predictions is very small (the size of the UDF is only $3.3 \times 10^{-3} \mathrm{deg}^{2}$ ), i.e., we do not expect the presence of a significant population of bright sources in the field. Second, we are working with an optically detected sample, and the bright submillimeter counts are dominated by optically thick sources that are likely not detected in the optical. For example, LESSJ0333243.6-274644, the only submillimeter source detected in the Hubble UDF as part of the LESS survey (LABOCA observations of the ECDF-S), with a flux density of $6.4 \mathrm{mJy}$ at $870 \mu \mathrm{m}$ (Weiß et al. 2009), has no optical counterpart in our catalog, presumably because it is an optically thick SMG (cf. Dunlop 2011). We find that the number counts at fluxes $\gtrsim 0.5 \mathrm{mJy}$ are dominated by galaxies with $\mu \hat{\tau}_{V}>1$, i.e., where the ISM is optically thick on average.

As an additional test on our number count predictions, we turn to a wider field covered by the LESS survey. To do so, we expand our analysis to the FIREWORKS photometric catalog (Wuyts et al. 2008) on the CDF-S, described in Section 2.2. FIREWORKS covers an area that is about 10 times the area of the UDF and about 10 times smaller than the full ECDF-S. The photometric catalog is much shallower than that for the UDF. For the area covered by FIREWORKS, we estimate between 6 and 23 sources to have $870 \mu \mathrm{m}$ fluxes above $4.7 \mathrm{mJy}$ (the flux limit of the LESS catalog). For comparison, Weiß et al. (2009) find 10 sources over the same area. Our prediction is thus broadly consistent with the LESS measurements.

\subsection{Extragalactic Background Light}

We can also compare our predictions with measurements of the integrated EBL in the submillimeter. Using our medianlikelihood estimate of the $345 \mathrm{GHz}$ flux density for each galaxy in the Hubble UDF, we obtain an integrated continuum $870 \mu \mathrm{m}$ flux density of $45.6 \mathrm{Jy} \mathrm{deg}^{-2}$. If we add the contribution from LESSJ0333243.6-274644 (which is not part of our sample 

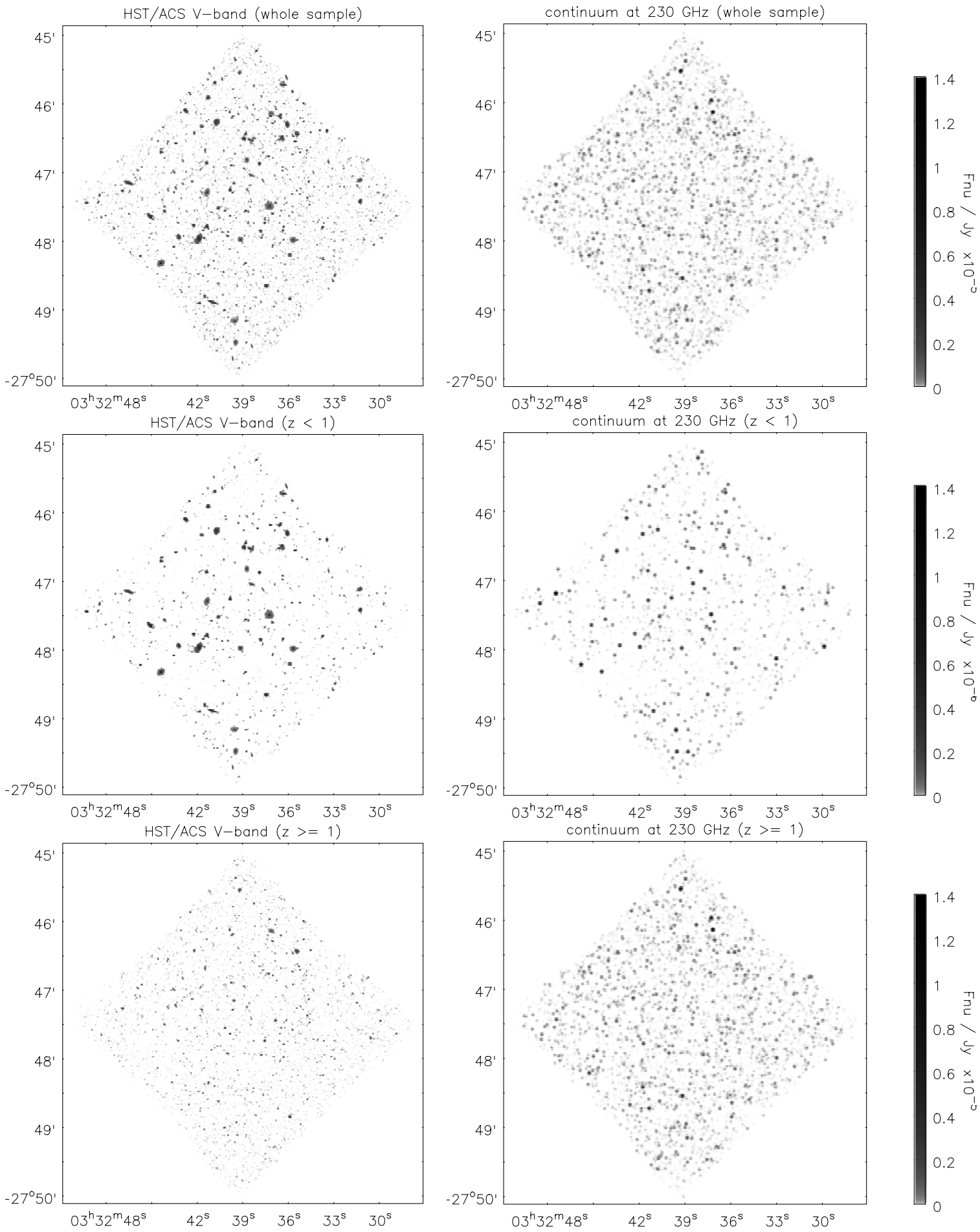

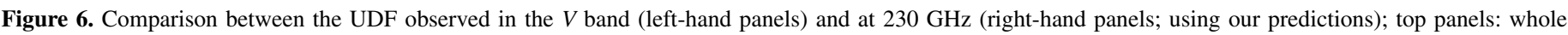

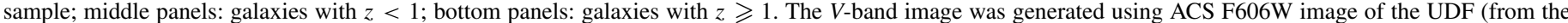

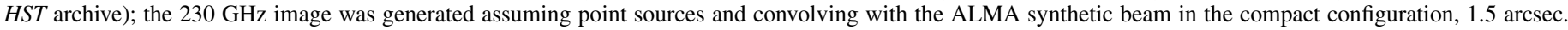

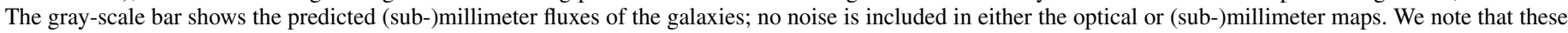
maps do not include the SMG galaxy detected in the UDF as part of the LESS survey (LESSJ0333243.6-274644).

but is detected in the LESS survey with a $6.4 \mathrm{mJy}$ flux), we obtain an EBL value of $47.5 \mathrm{Jy} \mathrm{deg}^{-2}$. This value is fully consistent with measurements of the EBL from $C O B E$ observations $44 \pm 15 \mathrm{Jy} \mathrm{deg}^{-2}$ (Puget et al. 1996; Fixsen et al. 1998). We note that the use of fixed SED templates to derive the (sub-)millimeter continuum fluxes of the galaxies would lead to EBL values that are inconsistent with the observed value (see
Appendix A). In Table 1, we list our estimates of the EBL in different (sub-)millimeter bands.

The estimated EBL at $870 \mu \mathrm{m}$ using the FIREWORKS catalog over the CDF-S area is $36.3 \mathrm{Jy} \mathrm{deg}^{-2}$, broadly consistent with our estimate based on the UDF area only (Table 1). This is lower than the $C O B E$ observed value quoted above, but it is still consistent with the observations, since the FIREWORKS 


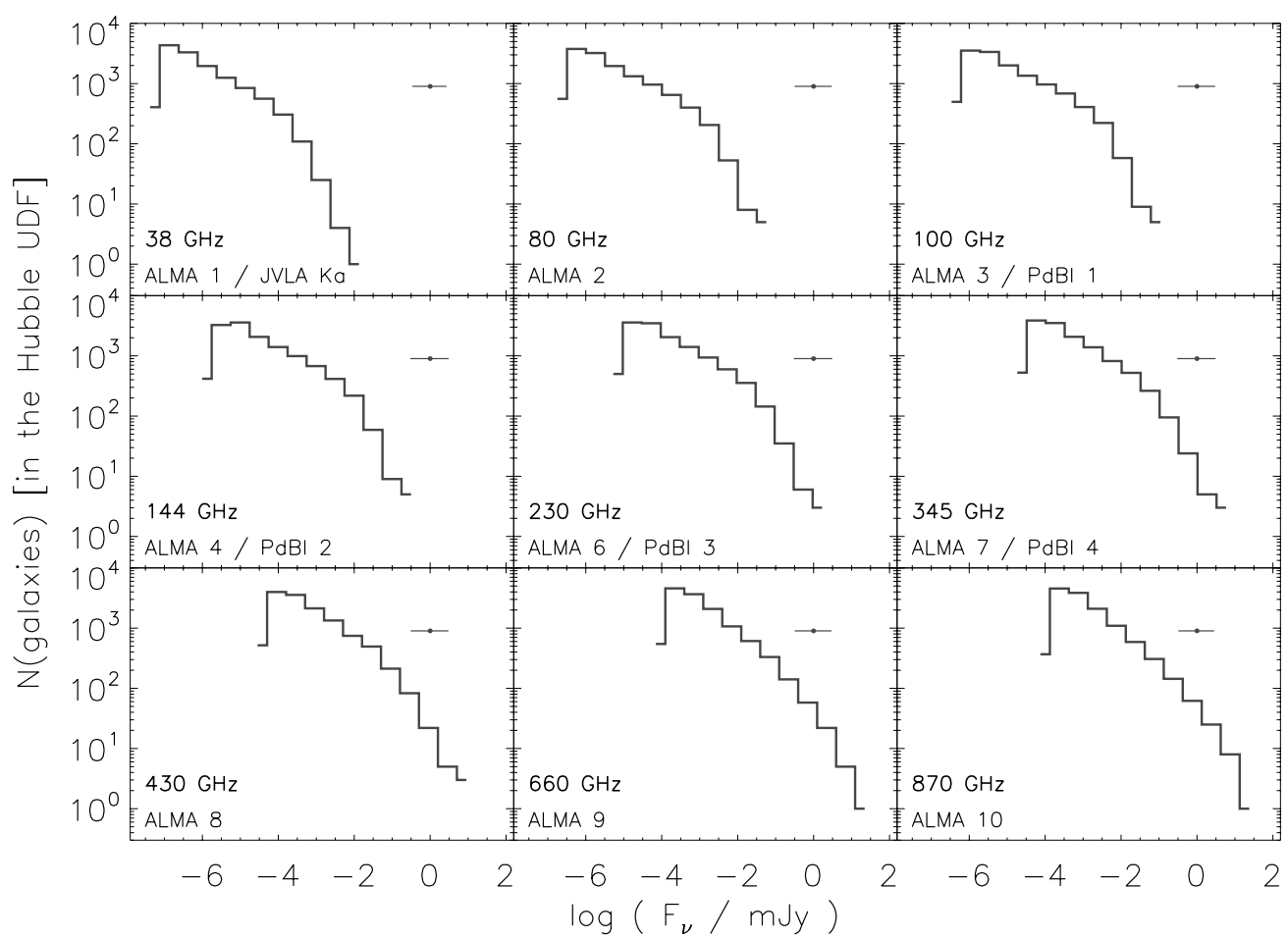

Figure 7. Distribution of the predicted continuum flux densities per flux density bin in different (sub-)millimeter bands for all the galaxies in our sample. The frequency is indicated in the bottom-left corner of each panel, as well as the corresponding bands in different observatories. The median confidence range for the continuum flux in each band is plotted in the upper right-hand corner of each panel.

Table 1

Predicted Extragalactic Background Light

\begin{tabular}{lcc}
\hline \hline $\begin{array}{l}\text { Frequency } \\
(\mathrm{GHz})\end{array}$ & Observatory & $\begin{array}{c}\text { EBL } \\
\left(\mathrm{Jy} \mathrm{deg}^{-2}\right)\end{array}$ \\
\hline 38 & ALMA 1, JVLA $\mathrm{Ka}$ & 0.10 \\
80 & ALMA 2 & 0.74 \\
100 & ALMA 3, PdBI/NOEMA 1 & 1.53 \\
144 & ALMA 4, PdBI/NOEMA 2 & 4.48 \\
230 & ALMA 6, PdBI/NOEMA 3 & 18.2 \\
345 & ALMA 7, PdBI/NOEMA 4 & 45.6 \\
430 & ALMA 8 & 63.1 \\
660 & ALMA 9 & 123 \\
870 & ALMA 10 & 146 \\
\hline
\end{tabular}

Notes. Estimates of the extragalactic background light at different frequencies using our flux predictions (area of the UDF field is $0.0033 \mathrm{deg}^{2}$ ) for the 13,099 galaxies in our sample.

catalog does not reach very deep, and therefore it is likely to miss the large number of faint galaxies that make up for a significant fraction of the EBL.

\subsection{Continuum Flux Density Predictions}

In this section, we present our general predictions for the continuum flux densities of our galaxies. In Table 6, we make our continuum predictions in all relevant (sub-)millimeter bands available for all the galaxies in our sample.

As an example, in Figure 6, we plot continuum map of the UDF at $230 \mathrm{GHz}$ (ALMA band 6, PdBI/NOEMA band 3) using our predictions (right-hand panels). Such images can be directly compared to future deep fields performed with ALMA or other facilities. We note that this figure is based solely on our optically based predictions, and so they are missing the only known bright (sub-)millimeter source in the UDF: LESSJ0333243.6-274644. The top panels of Figure 6 show our full sample, and we then divide the sample in two redshift bins $(z<1$; middle panels) and $(z \geqslant 1$; bottom panels). This illustrates the differences between galaxy detections as a function of redshift between the optical and the (sub-)millimeter, in particular that we expect galaxies to be relatively brighter in the (sub-)millimeter at high redshift thanks to the negative $k$-correction. Therefore, we will be able to detect "normal" galaxies out to higher redshifts in the (sub-)millimeter with new facilities, as we discuss in more detail in Section 5.

In Figure 7, we plot the distribution of the predicted continuum flux densities of all the galaxies in our sample in all current and future ALMA, JVLA, and IRAM PdBI/NOEMA bands from $38 \mathrm{GHz}$ to $870 \mathrm{GHz}$. We plot the expected number of galaxies per flux bin in the total UDF area in each band. The distribution of fluxes peaks at higher fluxes in the highest frequency ALMA bands, because, even taking into account $k$-correction effects, these bands sample the emission from the galaxies closer to the peak of the dust SED. In Section 5, we discuss the feasibility of performing a blind survey of the UDF with ALMA at full operation, and use these predicted fluxes, combined with the projected ALMA sensitivities, to obtain an estimate of the expected number of continuum detections.

\section{7. $\mathrm{CO}$ and [C II] Line Predictions}

ALMA and JVLA will detect $\mathrm{CO}$ and [C II] line emission from high-redshift galaxies, which will allow us to determine redshifts, molecular gas reservoirs, and dynamical masses (e.g., Solomon \& Vanden Bout 2005; Daddi et al. 2010b; Genzel et al. 2010; Tacconi et al. 2010; Walter et al. 2011). The rest-frame frequency, $v_{\text {rest }}$, of the $\mathrm{CO}$ lines corresponding to $J \rightarrow J-1$ transitions from $J=1$ to $J=7$ and of the [C II] line are given in Table 5. The observed frequency of each line varies with redshift as $v_{\text {obs }}=v_{\text {rest }}(1+z)^{-1}$. In Figure 14 (Appendix B), we plot the 

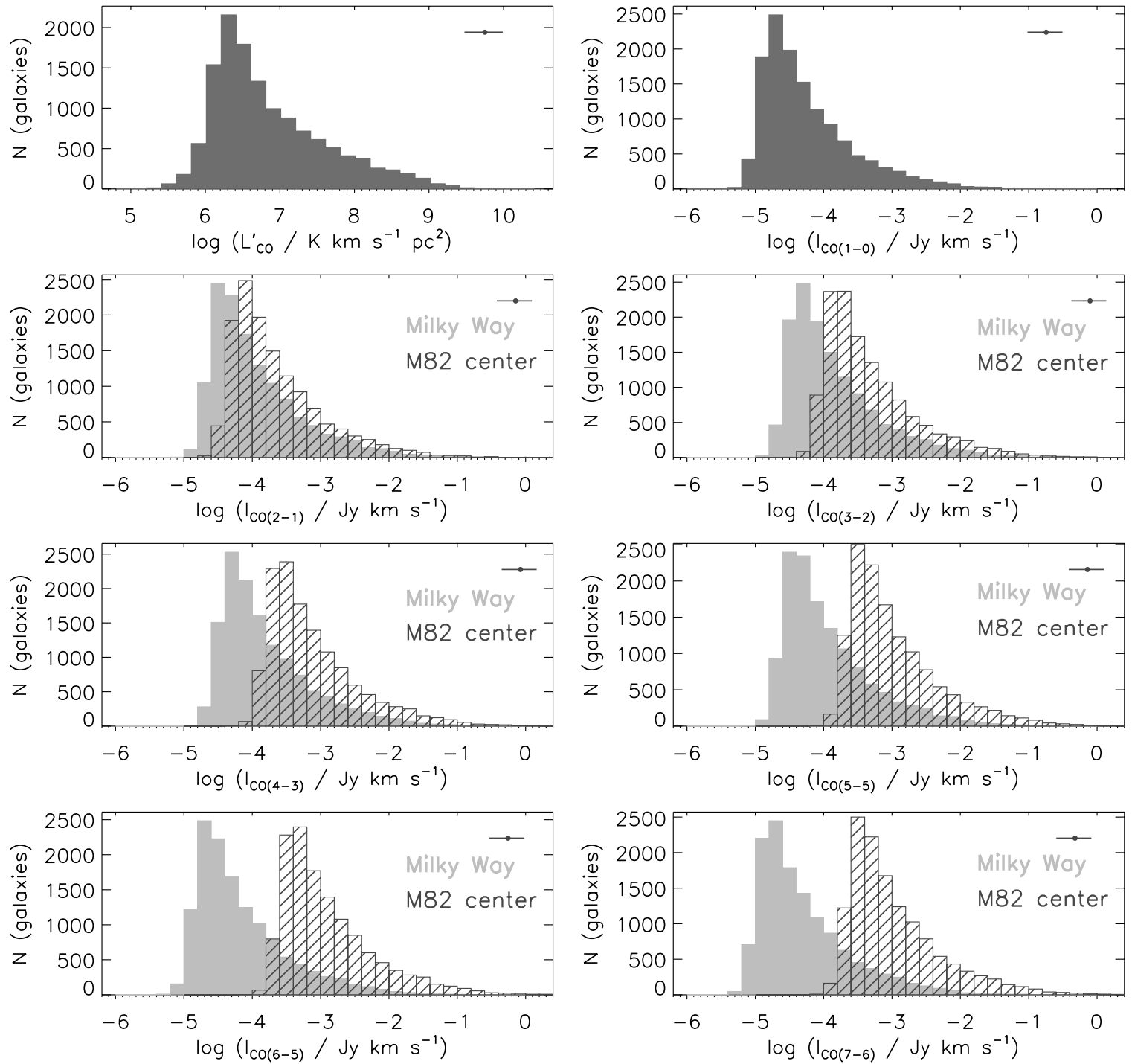

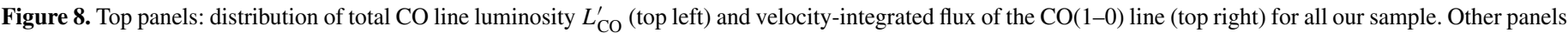

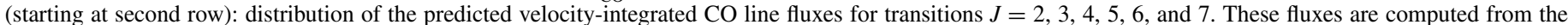

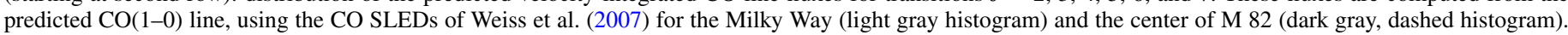
These two extreme CO SLEDs should bracket a large realistic range of possible CO excitations of star-forming galaxies (e.g., Weiss et al. 2007).

observed frequency of the seven first $\mathrm{CO}$ transitions, $\mathrm{CO}(1-0)$ (i.e., $J=1$ ) to $\mathrm{CO}(7-6)$ (i.e., $J=7$ ) and of the [C II] line as a function of redshift, with the frequency ranges covered by each ALMA, PdBI/NOEMA, and JVLA band shaded in gray and green. In Table 5 (Appendix B), we explicitly list the redshift ranges where the $\mathrm{CO}$ lines and $[\mathrm{C} \mathrm{II}]$ are observable in each band. It is clear that the lowest frequency bands, such as JVLA $K, K a$, and $Q$ will be crucial to probe the low $J \mathrm{CO}$ transitions, in particular the $\mathrm{CO}(1-0)$ line, in $z>1$ galaxies. All the other PdBI/NOEMA and ALMA bands will potentially detect higher $J \mathrm{CO}$ transitions at different redshifts, depending on the excitation state of the gas in galaxies. The highest frequency ALMA bands will not only sample high- $J$ CO lines at low redshifts and the continuum dust emission nearest to its peak, as mentioned in the previous section, but also the [C II] line out to high redshifts.

In this section, we attempt to predict the $\mathrm{CO}$ and $[\mathrm{C}$ II] line fluxes for the galaxies in our UDF sample using empirical relations that relate line luminosities with the infrared luminosity of the galaxies, for which we have a statistical estimate from our SED fits (Section 4.1).

\subsubsection{CO Emission}

The CO line luminosity of galaxies depends on various factors, such as the gas heating by starbursts, AGNs, and the cosmic microwave background at high redshifts (e.g., Combes et al. 1999; Obreschkow et al. 2009b; E. da Cunha et al., in preparation), as well as the clumpiness and metallicity of the gas (e.g., Obreschkow et al. 2009b). In this section, for simplicity, we predict the $\mathrm{CO}$ line luminosity of the galaxies in our sample using simple, empirically calibrated prescriptions. It has been found for a wide range of galaxy types, both in the local and highredshift Universe, that the CO line luminosity of star-forming galaxies correlates with their infrared luminosity (e.g., Solomon \& Vanden Bout 2005; Genzel et al. 2010; Daddi et al. 2010a).

The following relation between CO line luminosity and farinfrared luminosity was derived by Daddi et al. (2010a) for BzK galaxies, i.e., gas-rich star-forming disks at high redshifts:

$$
\log \left(L_{\mathrm{IR}}\right)=1.13 \log \left(L_{\mathrm{CO}}^{\prime}\right)+0.53,
$$

where $L_{\mathrm{IR}}$ is the infrared luminosity (integrated between 8 and $1000 \mu \mathrm{m})$ in $L_{\odot}$ and $L_{\mathrm{CO}}^{\prime}$ is the $\mathrm{CO}(1-0)$ line luminosity in 

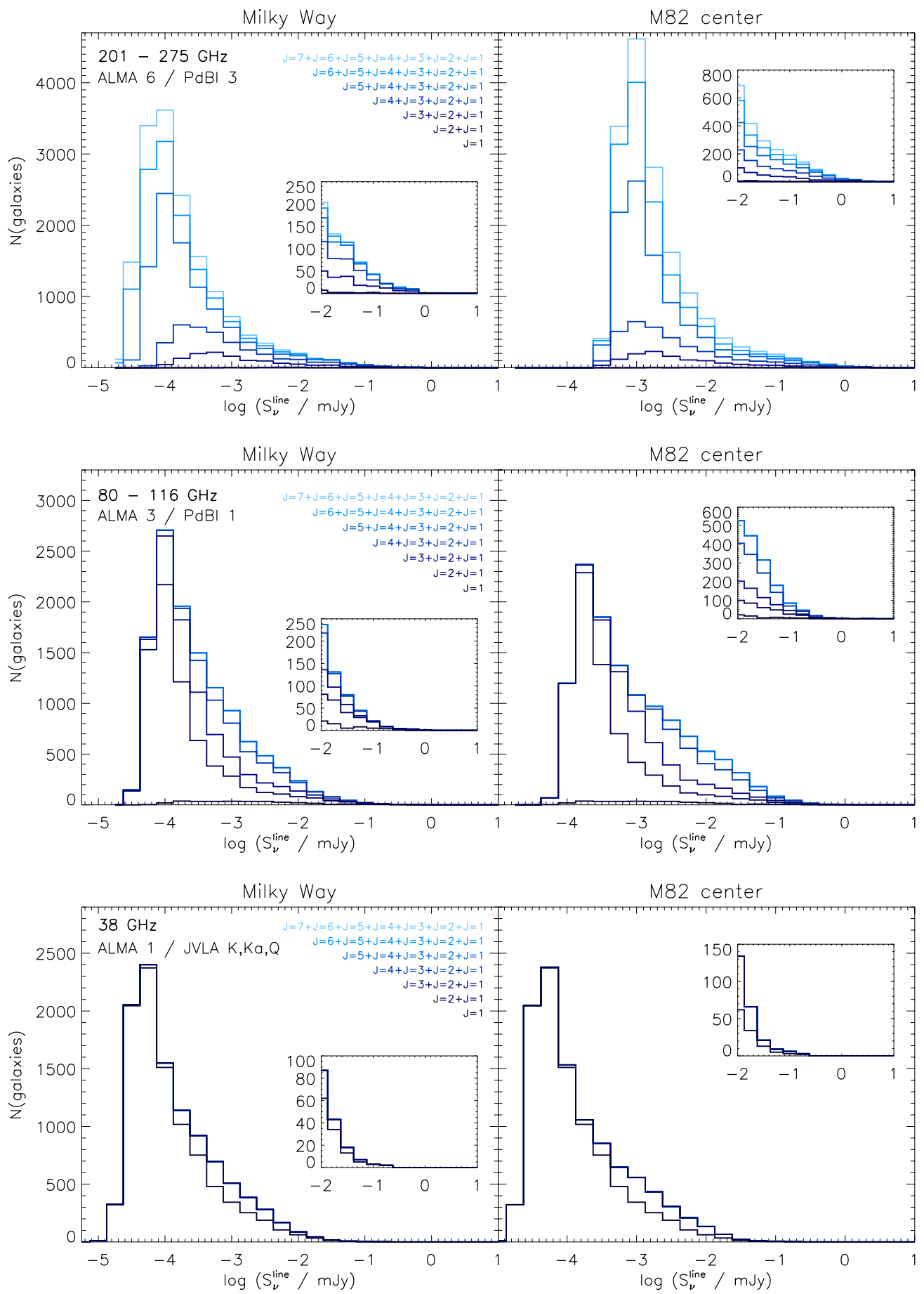

Figure 9. Number of expected $\mathrm{CO}$ line detections per line flux bin for three frequency ranges and two different molecular gas excitation scenarios. The line fluxes are computed assuming a line width of $300 \mathrm{~km} \mathrm{~s}^{-1}$. Top: frequency between 201 and $275 \mathrm{GHz}$ (ALMA band 6 and PdBI/NOEMA band 3); middle: frequency between 80 and $116 \mathrm{GHz}$ (ALMA band 3 and PdBI/NOEMA band 1); bottom: frequency between 18 and $50 \mathrm{GHz}$ (ALMA band 1 and JVLA bands $K, K a$, and $Q$ ). The left-hand and right-hand panels assume a Milky Way and M82 CO spectral line energy distribution, respectively. The lowest, darkest color histograms show the distribution of $\mathrm{CO}(1-0)$ fluxes, the next (lighter-colored) histogram shows the joint distribution of $\mathrm{CO}(1-0)$ and $\mathrm{CO}(2-1)$ fluxes, i.e., the distribution of $\mathrm{CO}(2-1)$ can be inferred from the increment in the histogram relatively to the histogram below, and so on until the lightest-colored histogram, which shows the sum of the distributions of all the CO line fluxes from $J=1$ to $J=7$.

(A color version of this figure is available in the online journal.)

$\mathrm{K} \mathrm{km} \mathrm{s}^{-1} \mathrm{pc}^{2}$. We obtain an estimate of $L_{\mathrm{CO}}^{\prime}$ using this equation and the statistical estimate on $L_{\mathrm{IR}}$ obtained for the SED fits of our galaxies; the resulting distribution of $L_{\mathrm{CO}}^{\prime}$ for the whole sample is plotted in the top left-hand panel of Figure 8. We chose this empirical calibration between $L_{\mathrm{IR}}$ and $L_{\mathrm{CO}}^{\prime}$ because our physical parameter estimates in Section 4.2 indicate that most of these galaxies would be comparable to normal, "mainsequence" star-forming disks, with typical infrared luminosities $L_{\mathrm{IR}} \lesssim 10^{11} L_{\odot}$. We note that Equation (1) is similar to the relation found by Genzel et al. (2010) for isolated, star-forming galaxies out to $z=2$. Other calibrations of this relation have been derived which include more extreme galaxies such as 

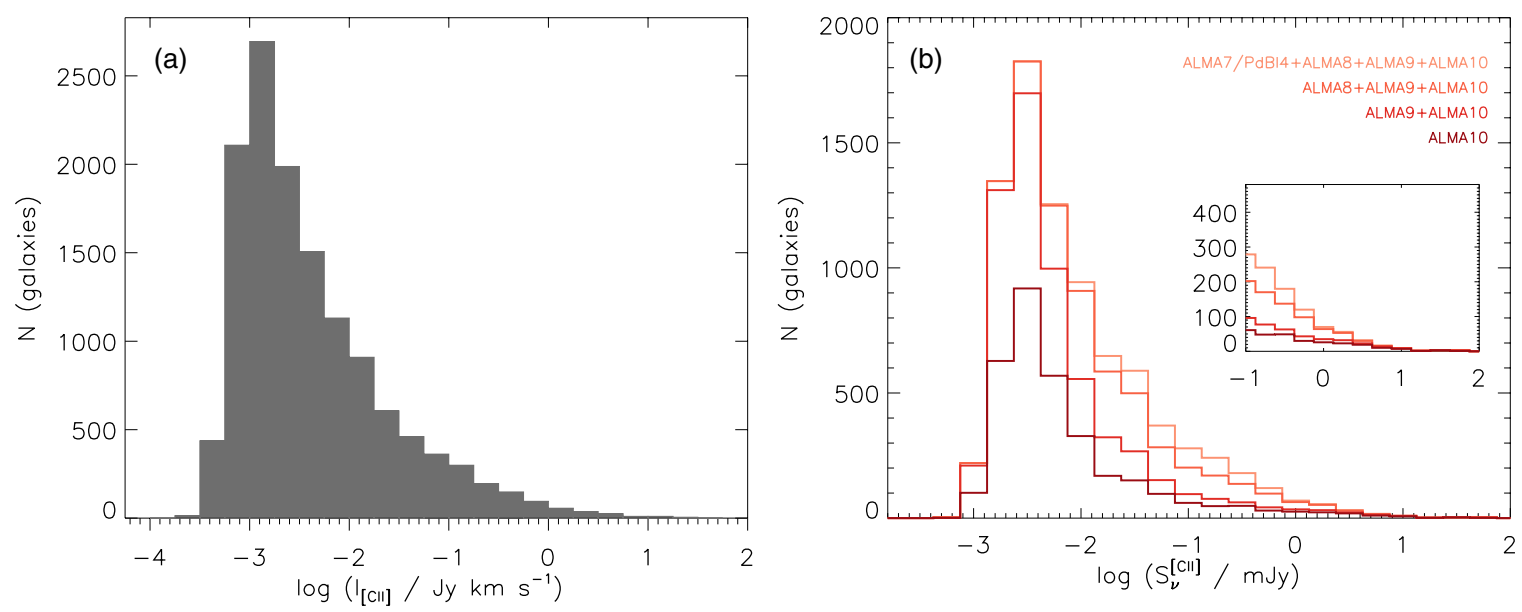

Figure 10. (a) Distribution of the velocity-integrated flux of the $\left[\mathrm{C}_{\mathrm{II}}\right]$ line, $I_{[\mathrm{CI}}$, for all the galaxies in our sample. (b) Distribution of the number of detections as a function of line flux, $S_{v}^{[\mathrm{C} \text { II] }}$ (computed assuming a $300 \mathrm{~km} \mathrm{~s}^{-1}$ line width), expected in the highest frequency bands available for ALMA and PdBI/NOEMA.

(A color version of this figure is available in the online journal.)

starbursts, mergers, and AGNs (e.g., Solomon \& Vanden Bout 2005; Riechers et al. 2006). When including these extreme galaxies, the relation between infrared and CO line luminosity becomes steeper, e.g., Solomon \& Vanden Bout (2005) find $\log \left(L_{\mathrm{FIR}}\right)=1.7 \log \left(L_{\mathrm{CO}}^{\prime}\right)-5.0$. Using this steeper relation for the infrared luminosity range of our galaxies $\left(L_{\text {dust }} \lesssim 10^{11} L_{\odot}\right)$ would result in $\mathrm{CO}$ line luminosities over one order of magnitude higher than those predicted using Equation (1) for the $L_{\mathrm{IR}}$ range of the galaxies in our sample. We discuss the implications of using these different assumptions for the predicted number of CO line detections in Section 5.

From $L_{\mathrm{CO}}^{\prime}$ (computed using Equation (1)), we then compute the corresponding flux of the $\mathrm{CO}(1-0)$ line, $S_{v}^{\mathrm{CO}(1-0)}$, using (e.g., Solomon \& Vanden Bout 2005):

$$
L_{\mathrm{CO}}^{\prime}=3.25 \times 10^{7} S_{v}^{\mathrm{CO}(1-0)} \Delta v v_{\mathrm{obs}}^{-2} D_{L}^{2}(1+z)^{-3},
$$

where $S_{v}^{\mathrm{CO}(1-0)}$ is the flux density in $\mathrm{Jy}, \Delta v$ is the line width in $\mathrm{km} \mathrm{s}^{-1}$ (the velocity-integrated flux of the line is $I_{\mathrm{CO}(1-0)}=$ $\left.S_{v}^{\mathrm{CO}(1-0)} \Delta v\right), v_{\mathrm{obs}}$ is the observed frequency of the line in $\mathrm{GHz}$, and $D_{L}$ is the luminosity distance in Mpc. We assume a typical line width of $300 \mathrm{~km} \mathrm{~s}^{-1}$, consistent with typical line widths measured in high-redshift star-forming galaxies (e.g., Daddi et al. 2010a; Genzel et al. 2010; Tacconi et al. 2010). In the top right-hand panel of Figure 8, we plot the distribution of the velocity-integrated flux of the $\mathrm{CO}(1-0)$ line for all the galaxies in our sample computed using Equation (2).

The fluxes of higher transition $\mathrm{CO}$ lines depend highly on the excitation of the $\mathrm{CO}$ gas in galaxies. Different physical conditions in the gas produce different $\mathrm{CO}$ spectral line energy distributions (SLEDs; e.g., Weiss et al. 2007), which translate into different ratios between the $\mathrm{CO}(1-0)$ line and the higher $J$ lines. To compute the predicted flux of the $\mathrm{CO}(2-1), \mathrm{CO}(3-2)$, $\mathrm{CO}(4-3), \mathrm{CO}(5-4), \mathrm{CO}(6-5)$, and $\mathrm{CO}(7-6)$ lines, we assume two possible CO SLEDs from Weiss et al. (2007): the Milky Way CO SLED and the M 82 center CO SLED. These two cases correspond to very low and high excitation of the gas, respectively, and should bracket a large realistic range of possible physical conditions in star-forming galaxies. In the six bottom panels of Figure 8, we show the distribution of expected velocity-integrated $\mathrm{CO}$ line fluxes for the galaxies in our sample, and compare the predictions for these two excitation scenarios. For each $\mathrm{CO}$ line, these two extreme excitations should bracket the full range of line fluxes expected for our sample of starforming galaxies (as supported by observations of multiple $\mathrm{CO}$ lines in a wide range of systems from local quiescent galaxies to high-redshift QSOs; see, e.g., Weiss et al. 2007). The difference between the predictions of $\mathrm{CO}$ line fluxes using these two $\mathrm{CO}$ SLEDs increases with increasing $J$ : the higher $J$ CO lines are stronger in the case of the M 82 center SLED, which corresponds to a higher $\mathrm{CO}$ excitation. In Table 7, we provide the predicted CO line fluxes for all the galaxies in our sample (including both $\mathrm{CO}$ excitation scenarios).

To predict the number of expected $\mathrm{CO}$ line detections given a certain flux limit in various (sub-)millimeter bands (ALMA, JVLA, or PdBI/NOEMA), we can build the expected distribution of $\mathrm{CO}$ line fluxes in each band based on the predictions described above. First, for each frequency band listed in Table 5, we retain galaxies for which the redshift falls in a range where one of the $\mathrm{CO}$ lines can be observed (these ranges are listed in Table 5, see also Figure 14). Then, we compute the expected

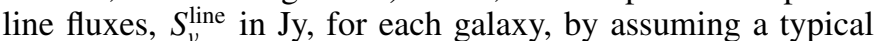
line width of $\Delta v=300 \mathrm{~km} \mathrm{~s}^{-1}$ (using Equation (2) to the get flux of the $\mathrm{CO}(1-0)$ line and the CO SLEDs to get the higher $J$ lines). In Figure 9, as examples, we plot the distribution of line fluxes from $\mathrm{CO}(1-0)$ to $\mathrm{CO}(7-6)$ if one were to fully cover the bands from 201 to $275 \mathrm{GHz}$ (ALMA band 6, PdBI/NOEMA band 3), 80-116 GHz (ALMA band 3, PdBI/NOEMA band 1), and $18-50 \mathrm{GHz}$ (ALMA band 1/JVLA bands $K, K a$, and $Q$ ). We show the $\mathrm{CO}$ line fluxes corresponding to two gas excitation scenarios (i.e., CO SLEDs): Milky Way type (left-hand panels) and M82-type (right-hand panels). For example, in the frequency range $80-116 \mathrm{GHz}$, we plot the distribution of $\mathrm{CO}(1-0)$ line fluxes only of galaxies with redshifts $z \leqslant 0.44$, for which the $\mathrm{CO}(1-0)$ line would be redshifted to that frequency band (Table 5). Similarly, for the distribution of $\mathrm{CO}(2-1)$ line fluxes in that band, we include only galaxies with $0.99 \leqslant z \leqslant 1.88$, and so on until the $\mathrm{CO}(7-6)$ line. For clarity, the distributions plotted in Figure 9 are cumulative: the darkest-colored histogram shows the number of galaxies per line flux bin for the $\mathrm{CO}(1-0)$ line, the next, lighter histogram shows the number of galaxies per line flux bin for the $\mathrm{CO}(1-0)$ and $\mathrm{CO}(2-1)$ line, the next histogram adds the number of galaxies per line flux bin for the $\mathrm{CO}(3-2)$ line, etc. That is, the lightest-colored histogram shows the total number of galaxies per line flux bin when including all $\mathrm{CO}$ lines from $J=1$ to $J=7$. In general, Figure 9 shows that 

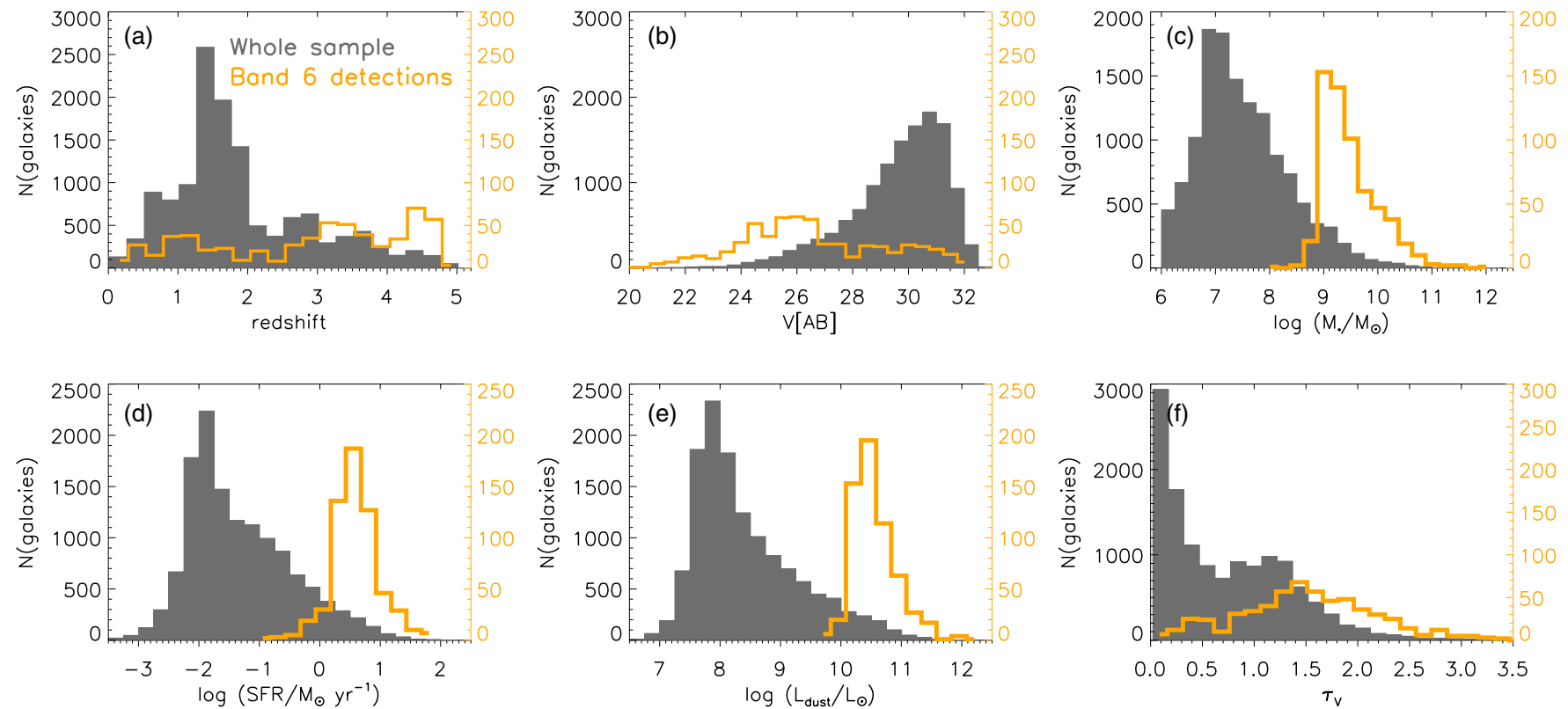

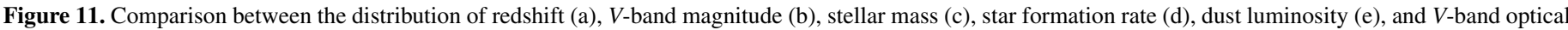

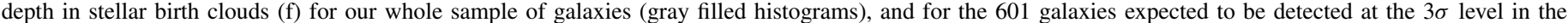

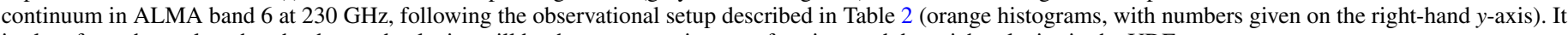
is clear from these plots that the detected galaxies will be the most massive, star-forming, and dust-rich galaxies in the UDF.

(A color version of this figure is available in the online journal.)

the number of galaxies in the highest flux bins is largest if the CO SLED is M82-like, as expected, so the number of detections given a certain flux limit greatly depends on the $\mathrm{CO}$ excitation (as discussed in Section 5.2).

\subsection{2. [C II] Emission}

The [C II] line at $158 \mu \mathrm{m}$ is the main cooling line of the ISM in galaxies, and it arises mainly from photo-dissociation regions - at the interface between the ionized gas and the neutral and molecular gas-which are typically associated with starforming regions. This line is therefore one of the main farinfrared tracers of star formation in galaxies (Stacey et al. 1991, 2010; Boselli et al. 2002; de Looze et al. 2011). Since this is the brightest far-infrared line, it will be readily detected in deep observations with ALMA, particularly using the highest frequency bands.

We rely on previous observational studies of the ratio of [C II] line to far-infrared luminosity $\left(L_{[\mathrm{CI}} / L_{\mathrm{FIR}}\right)$ of galaxies to estimate the [C II] line luminosity for each galaxy in our sample. The ratio $L_{[\mathrm{CI}]} / L_{\mathrm{FIR}}$ of normal star-forming galaxies varies typically between $1 \%$ and $0.1 \%$, and has been shown to anti-correlate with dust heating intensity (e.g., Brauher et al. 2008). Here, for simplicity, and considering the relatively large error bars on our $L_{\text {FIR }}$ estimates, we adopt a constant ratio of $\log \left(L_{\left[\mathrm{C}_{\mathrm{II}}\right]} / L_{\mathrm{FIR}}\right)=-2.5$, which corresponds to the average value for normal star-forming galaxies with low far-infrared luminosities $L_{\mathrm{IR}} \lesssim 10^{11} L_{\odot}$ (i.e., similar to the galaxies in our sample) and average dust heating (Boselli et al. 2002; Brauher et al. 2008; Graciá-Carpio et al. 2011; Cox et al. 2011). In Figure 10(a), we plot the distribution of the expected velocityintegrated flux of [C II],$I_{[\mathrm{CI}]}=S_{v}^{[\mathrm{CI}]} \Delta v$ for all the galaxies in our sample, computed using Equation (2):

$$
L_{[\mathrm{C} \text { II }]}=1.04 \times 10^{-3} S_{v}^{[\mathrm{C} \text { II] }} \Delta v v_{\text {rest }} D_{L}^{2}(1+z)^{-1},
$$

where $L_{\left[\mathrm{C}_{\mathrm{II}}\right]}$ is the [C II] line luminosity in $L_{\odot}, S_{v}^{[\mathrm{CII}]}$ is the line flux in Jy, $\Delta v$ is the velocity dispersion in $\mathrm{km} \mathrm{s}^{-1}, D_{L}$ is the luminosity distance, and $v_{\text {rest }}=1900.54 \mathrm{GHz}$. At $z<5$, the observed frequency of the [C II] line falls in the highest frequency ALMA bands, namely, bands 7, 8, 9, and 10 (Figure 14). In Figure 10(b), we plot the distribution of expected detections in these bands as a function of [C II] line flux (computed assuming $\Delta v=300 \mathrm{~km} \mathrm{~s}^{-1}$ ). In Table 7, we provide the predicted [C II] line fluxes for all the galaxies in our sample.

\section{POSSIBLE DEEP FIELD STRATEGIES WITH ALMA}

Based on the results presented in the previous sections, we now discuss the feasibility of carrying out a deep field survey of the Hubble UDF with ALMA at full operations (i.e., using 50 antennas). In the following, we will assume a total time of $500 \mathrm{hr}$ for the full survey, and investigate the possible setups of such a survey to maximize the redshift coverage and number of galaxy detections.

One immediate drawback of ALMA as a survey instrument is that the primary beam size, which is driven by the size of the antennas, is relatively small in all bands: the primary beam size is given, to the first order, by $20^{\prime \prime} .3 \times 300 /(v / \mathrm{GHz})$, where $v$ is the observing frequency. Therefore, even a relatively small area field as the Hubble UDF $\left(3{ }^{\prime} .45 \times 33^{\prime} .45\right.$, i.e., a total area of $11.9 \operatorname{arcmin}^{2}$ ), will be hard to cover with ALMA, and will require significant mosaicking. It is beyond the scope of this paper to go into the technical details of how such a mosaic should be set up. In the following, for illustration purposes, we simply consider a total on-source time of $500 \mathrm{hr}$ for the UDF, which we divide in different pointings to cover the area of the UDF-we do not include overheads and mosaicking details (such as degrading sensitivities inside the beam, etc.).

\subsection{Continuum Detections}

For each ALMA band, given the area of the primary beam, we compute how many effective pointings are needed to cover 
Table 2

Summary of a Possible Observational Setup to Observed the Hubble UDF with ALMA in Full Operations with a Total On-source Time of 500 hr

\begin{tabular}{|c|c|c|c|c|c|c|c|c|c|}
\hline Band & $\begin{array}{l}\text { Frequency } \\
\quad(\mathrm{GHz})\end{array}$ & $\begin{array}{c}\text { Primary Beam } \\
(\operatorname{arcsec})\end{array}$ & $\begin{array}{l}\text { Number of } \\
\text { Pointings }^{\mathrm{a}}\end{array}$ & $\begin{array}{l}\text { Number of } \\
\text { Frequency } \\
\text { Settings }\end{array}$ & $\begin{array}{l}\text { Time per } \\
\text { Pointing } \\
\text { (h) }\end{array}$ & $\begin{array}{c}\text { Time per } \\
\text { Frequency Setting } \\
\text { (h) }\end{array}$ & $\begin{array}{l}\sigma_{\mathrm{cont}^{\mathrm{c}}} \\
(\mathrm{mJy})\end{array}$ & $\begin{array}{l}\sigma_{\text {line }}{ }^{\mathrm{d}} \\
(\mathrm{mJy})\end{array}$ & $\begin{array}{l}\text { Number of } \\
3 \sigma \text { Continuum }^{\mathrm{e}} \\
\text { Detections }^{\mathrm{e}}\end{array}$ \\
\hline ALMA 1 & 38 & 140 & 3 & 2 & 167 & 83.5 & $3.7 \times 10^{-4}$ & $7.6 \times 10^{-3}$ & 36 \\
\hline ALMA 2 & 80 & 76 & 10 & 3 & 50 & 16.7 & $9.9 \times 10^{-4}$ & $1.7 \times 10^{-2}$ & 155 \\
\hline ALMA 3 & 100 & 62 & 15 & 4 & 33 & 8.25 & $1.5 \times 10^{-3}$ & $2.7 \times 10^{-2}$ & 221 \\
\hline ALMA 4 & 144 & 43 & 30 & 5 & 17 & 3.40 & $2.5 \times 10^{-3}$ & $4.2 \times 10^{-2}$ & 363 \\
\hline ALMA 6 & 230 & 26 & 81 & 8 & 6.2 & 0.78 & $5.1 \times 10^{-3}$ & $8.4 \times 10^{-2}$ & 601 \\
\hline ALMA 7 & 345 & 18 & 169 & 13 & 3.0 & 0.23 & $1.3 \times 10^{-2}$ & $2.3 \times 10^{-1}$ & 522 \\
\hline ALMA 8 & 430 & 14 & 278 & 15 & 1.8 & 0.12 & $8.0 \times 10^{-2}$ & $1.3 \times 10^{0}$ & 136 \\
\hline ALMA 9 & 660 & 9.3 & 630 & 14 & 0.8 & 0.06 & $2.4 \times 10^{-1}$ & $3.1 \times 10^{0}$ & 84 \\
\hline ALMA 10 & 870 & 1.1 & 1080 & 21 & 0.5 & 0.03 & $7.1 \times 10^{-1}$ & $9.5 \times 10^{0}$ & 40 \\
\hline
\end{tabular}

Notes. Sensitivities computed using the ALMA Sensitivity Calculator (50 antennas and default weather conditions).

a Computed by dividing the total UDF area by the area of the primary beam in each band.

${ }^{\mathrm{b}}$ Computed by dividing $500 \mathrm{hr}$ by number of pointings in each band.

c $8 \mathrm{GHz}$ bandwidth.

d $300 \mathrm{~km} \mathrm{~s}^{-1}$ bandwidth.

e Expected number of continuum detections over the whole UDF field for each band. The expected number of line detections is given in Table 3.

the whole area of the UDF (Table 2). The number of pointings needed to cover the UDF increases from band 1 to band 10 , from only 3 pointings to over 1000 pointings. In this backof-the-envelope calculation we take the integration time per pointing in each band as the total $500 \mathrm{hr}$ divided by the required number of pointings, and then use the ALMA Sensitivity Calculator (ASC) $)^{13}$ to compute the continuum sensitivity that can be reached in each integration time, assuming a $8 \mathrm{GHz}$ bandwidth and the default weather conditions. In Table 2, we list the sensitivities and the expected number of $3 \sigma$ continuum detections in each band for the whole field, based on our continuum predictions of Section 4.6. The resulting number of predicted continuum detections is a combination of the intrinsic flux of each galaxy, the $k$-correction for each galaxy at each redshift, and the changing integration time per pointing due to varying beam size; for reference, $1 \mathrm{hr}$ integration time corresponds to an rms of $4.81,12.6$, and $483 \mu \mathrm{Jy}$ at $38 \mathrm{GHz}$ (ALMA 1), $230 \mathrm{GHz}$ (ALMA 6), and $870 \mathrm{GHz}$ (ALMA 10), respectively. Table 2 shows that the number of expected continuum detections is maximum for band 6 at $230 \mathrm{GHz}$ (601 detections), with a large number of predicted detections also in band 3 at $100 \mathrm{GHz}(221)$, band 4 at $144 \mathrm{GHz}$ (363), and band 7 at $345 \mathrm{GHz}$ (522). In Figures 1 and 11, we compare the distributions of the properties of the 601 galaxies that would be detected at the $3 \sigma$ level in the ALMA band 6 to those of the original sample. We note that the redshift distribution of these sources is relatively flat, thanks to the negative $k$-correction in the submillimeter. Also not surprisingly, these figures show that we expect to detect only the most massive, highly star-forming, and dusty galaxies in our sample. These galaxies are still over an order of magnitude star-forming and dusty than classic SMGs detected in blind "pre-ALMA" (sub-)millimeter surveys, which typically have SFRs $\gtrsim 100 M_{\odot} \mathrm{yr}^{-1}$ and dust luminosities $\gtrsim 10^{12} L_{\odot}$. The median detected galaxy has a stellar mass of $3 \times 10^{9} M_{\odot}$, an SFR of $\sim 5 M_{\odot} \mathrm{yr}^{-1}$, dust luminosity of $4 \times 10^{10} L_{\odot}$, dust mass of $5 \times 10^{7} M_{\odot}$, and $V$-band effective optical depth in stellar birth clouds of 1.6. This implies that the typical detected galaxy would be about 100 times more star-forming, more massive (in terms of stellar content), and more dusty, and about 10 times

\footnotetext{
13 http://almascience.eso.org/call-for-proposals/sensitivity-calculator
}

more obscured in the optical than the median of the whole UDF sample.

\subsection{Line Detections}

We now discuss the possibility of detecting $\mathrm{CO}$ and $[\mathrm{C} \mathrm{II}]$ lines in the UDF using frequency scans in all ALMA bands. We compute the expected line sensitivities for a total on-source time of $500 \mathrm{hr}$ on the UDF as in Section 5.1, but taking into account the time needed to scan in band in frequency space. The frequency interval covered in each frequency setting is $\Delta v=8 \mathrm{GHz}$. Therefore, the total number of settings required to scan a given ALMA band is the total frequency range of the band divided by $\Delta v$ (we note that the exact setup will depend on the sideband separations in the various bands). The total time spent in each frequency setting is then the integration time per pointing divided by the required number of settings in each band. We assume the same mosaicking scheme of the UDF and use the same integration time per pointing in each band as for the continuum (Section 5.1). The total number of frequency settings, time per setting, and resulting line sensitivity $\sigma_{\text {line }}$ (computed using the ASC and assuming a bandwidth of $300 \mathrm{~km} \mathrm{~s}^{-1}$ in order to resolve the lines in velocity space) are listed in Table 2 . In Table 3, we show the predicted number of $5 \sigma$ line detections in each band given the line sensitivities $\sigma_{\text {line }}$ of Table 2, assuming both the Milky Way and the M 82 CO SLED, as in Section 4.7.1. It is clear that with the integration times per frequency setting of Table 2 we predict a relatively low number of line detections, compared with the predicted number of continuum detections. In bands 2-6, we predict a minimum of about $20 \mathrm{CO}$ line detections in each band, when assuming the Milky Way CO SLED; around 100 detections are predicted if the $\mathrm{CO}$ is more excited. To get more line detections, one would need to go deeper, which likely implies a compromise with the area of the survey. For example, to reach an rms of $10 \mu \mathrm{Jy}$ in band 6 (approximately 8 times deeper than reached in the "default" survey setup considered so far), which would yield at least between 200 and 800 detections over the whole UDF, one would need over $400 \mathrm{hr}$ of effective integration time for each of the 81 pointings.

As mentioned in Section 4.7.1, if we use the Solomon \& Vanden Bout (2005) calibration to convert the infrared luminosities of our galaxies into $\mathrm{CO}$ line luminosities, we 
Table 3

Predicted Number of $5 \sigma$ CO Line Detections in the Hubble UDF with ALMA

\begin{tabular}{lcc}
\hline \hline & ALMA $1(31.3-45 \mathrm{GHz})$ \\
$5 \sigma_{\text {line }}=3.82 \times 10^{-2} \mathrm{mJy}$ & \\
\hline Line & Milky Way & M 82 Center \\
\hline $\mathrm{CO}(1-0)$ & 17 & 17 \\
$\mathrm{CO}(2-1)$ & 5 & 16
\end{tabular}

\begin{tabular}{|c|c|c|}
\hline \multicolumn{3}{|c|}{$\begin{array}{c}\text { ALMA } 2(67-90 \mathrm{GHz}) \\
5 \sigma_{\text {line }}=8.55 \times 10^{-2} \mathrm{mJy}\end{array}$} \\
\hline Line & Milky Way & M 82 Center \\
\hline $\mathrm{CO}(1-0)$ & 21 & 21 \\
\hline $\mathrm{CO}(2-1)$ & 7 & 16 \\
\hline $\mathrm{CO}(3-2)$ & 1 & 38 \\
\hline $\mathrm{CO}(4-3)$ & 2 & 14 \\
\hline
\end{tabular}

\begin{tabular}{|c|c|c|}
\hline \multicolumn{3}{|c|}{$\begin{array}{l}\text { ALMA } 3(84-116 \mathrm{GHz}) \\
5 \sigma_{\text {line }}=1.34 \times 10^{-1} \mathrm{mJy}\end{array}$} \\
\hline Line & Milky Way & M 82 Center \\
\hline $\mathrm{CO}(1-0)$ & 5 & 5 \\
\hline $\mathrm{CO}(2-1)$ & 15 & 37 \\
\hline $\mathrm{CO}(3-2)$ & 0 & 17 \\
\hline $\mathrm{CO}(4-3)$ & 0 & 37 \\
\hline $\mathrm{CO}(5-4)$ & 0 & 11 \\
\hline \multicolumn{3}{|c|}{$\begin{array}{l}\text { ALMA } 4(125-163 \mathrm{GHz}) \\
5 \sigma_{\text {line }}=2.10 \times 10^{-1} \mathrm{mJy}\end{array}$} \\
\hline Line & Milky Way & M 82 Center \\
\hline $\mathrm{CO}(2-1)$ & 14 & 26 \\
\hline $\mathrm{CO}(3-2)$ & 4 & 27 \\
\hline $\mathrm{CO}(4-3)$ & 0 & 12 \\
\hline $\mathrm{CO}(5-4)$ & 0 & 32 \\
\hline $\mathrm{CO}(6-5)$ & 0 & 27 \\
\hline $\mathrm{CO}(7-6)$ & 0 & 8 \\
\hline & $\begin{array}{l}\text { A } 6(211-27 \\
=4.22 \times 10\end{array}$ & \\
\hline
\end{tabular}

\begin{tabular}{lcc}
\hline Line & Milky Way & M 82 Center \\
\hline $\mathrm{CO}(2-1)$ & 0 & 0 \\
$\mathrm{CO}(3-2)$ & 10 & 30 \\
$\mathrm{CO}(4-3)$ & 6 & 39 \\
$\mathrm{CO}(5-4)$ & 3 & 43 \\
$\mathrm{CO}(6-5)$ & 0 & 18 \\
$\mathrm{CO}(7-6)$ & 0 & 7 \\
\hline & ALMA $7(275-373 \mathrm{GHz})$ & \\
\hline $\mathrm{Line}$ & $5 \sigma_{\text {line }}=1.15 \times 10^{0} \mathrm{mJy}$ & 0 \\
\hline $\mathrm{CO}(3-2)$ & Milky Way & 22 \\
$\mathrm{CO}(4-3)$ & 0 & 30 \\
$\mathrm{CO}(5-4)$ & 0 & 22 \\
$\mathrm{CO}(6-5)$ & 0 & 10 \\
$\mathrm{CO}(7-6)$ & 0 & 22 \\
\hline
\end{tabular}

Note. Assuming a total on-source integration time of $500 \mathrm{hr}$, for two different cases of CO line SLEDs: Milky Way and M 82 center (Section 4.7.1).

would obtain intrinsically brighter $\mathrm{CO}$ lines by about one order of magnitude. This would make the number of predicted $\mathrm{CO}$ line detections in the observational setup discussed here much higher than the numbers listed in Table 3. In the bands with most predicted detections, ALMA 2 to ALMA 6, the minimum number of detections (when assuming the Milky Way SLED) would increase from on average 22 to 116 in each band, and the maximum number of detections (when assuming the M 82 SLED) would increase from on average 50 to 242 in each band.
Table 4

Predicted Number of $5 \sigma[\mathrm{C}$ II] Line Detections in the Hubble UDF with ALMA

\begin{tabular}{|c|c|c|c|}
\hline Band & $\begin{array}{l}\text { Frequency Range } \\
\qquad(\mathrm{GHz})\end{array}$ & $\begin{array}{l}5 \sigma_{\text {line }} \\
(\mathrm{mJy})\end{array}$ & $\begin{array}{l}\text { Number of } 5 \sigma \\
{\left[\mathrm{C}_{\mathrm{II}}\right] \text { Detections }}\end{array}$ \\
\hline ALMA 7 & $275-373$ & 1.15 & 10 \\
\hline ALMA 8 & $385-500$ & 6.65 & 0 \\
\hline ALMA 9 & $602-710$ & 15.6 & 3 \\
\hline ALMA 10 & $787-950$ & 47.3 & 2 \\
\hline
\end{tabular}

Note. Assuming a total on-source integration time of $500 \mathrm{hr}$.

We use the same method to estimate the number of expected [C II] line detections in the highest frequency ALMA bands, based on our estimates of [C II] line fluxes from Section 4.7.2. We predict a total of $15[\mathrm{C} \mathrm{II}] 5 \sigma$ detections using the integration times per pointing and per frequency setting in each band listed in Table 2 (Table 4). In bands 8-10, these numbers represent a low fraction $(\lesssim 0.1 \%)$ of the total number of galaxies in the observable redshift range because the large number of required pointings to cover the whole UDF implies a very short integration time per pointing and hence a relatively high rms. The minimum dust luminosities and SFRs of the galaxies that can be detected in [C II] using this observational setup are $5 \times 10^{11} L_{\odot}$ and $10 M_{\odot} \mathrm{yr}^{-1}$, at the very high end of the distribution for the whole sample (e.g., Figure 11). With band 7, the number of detections is higher and because the rms is smaller thanks to a higher integration time, allowing us to go deeper and detect $[\mathrm{C} \mathrm{II}]$ emission from galaxies with dust luminosities as low as $1.4 \times 10^{11} L_{\odot}$.

\section{DISCUSSION AND CONCLUSIONS}

In this paper, we have presented empirical predictions of (sub-)millimeter continuum and $\mathrm{CO} /[\mathrm{C} \mathrm{II}]$ line fluxes for a sample of 13,099 galaxies with redshifts up to $z=5$, selected in the deepest optical/near-infrared catalog of the Hubble UDF over $12 \operatorname{arcmin}^{2}$. We have performed a self-consistent modeling of the observed optical/near-infrared SEDs of the galaxies, which relies on an energy-balance technique, and have allowed us to obtain Bayesian estimates of the total infrared luminosity and (sub-)millimeter continuum flux densities in several ALMA, JVLA, and PdBI/NOEMA bands. We then combined the constraints on total infrared luminosity of our galaxies with empirical correlations to obtain estimates of their $\mathrm{CO}$ and $[\mathrm{C} \mathrm{II}]$ line luminosities. One advantage of our method is that it allows us to derive reliable confidence ranges for the physical parameters of the galaxies derived from SED fitting. Using fits to the complete ultraviolet to far-infrared SEDs of a sub-sample of galaxies in the UDF, we show that, even though our confidence ranges for the infrared luminosities and (sub-)millimeter continuum fluxes can be as large as one order of magnitude, our estimates are reliable, and the large confidence ranges reflect the inherent uncertainty in deriving infrared and (sub-)millimeter properties of galaxies from optical observations.

Our predictions rely mainly on two assumptions. First, that we can reliably predict the total infrared luminosity from the attenuated stellar emission, i.e., that our dust attenuation prescription is correct. We expect this to be true for moderately star-forming, optically selected galaxies with moderate dust optical depths and infrared luminosities (Charlot \& Fall 2000; da Cunha et al. 2008; Daddi et al. 2005, 2007; Reddy et al. 2006). Another argument in favor of the relatively low typical optical depths of our galaxies is that, if we assumed 


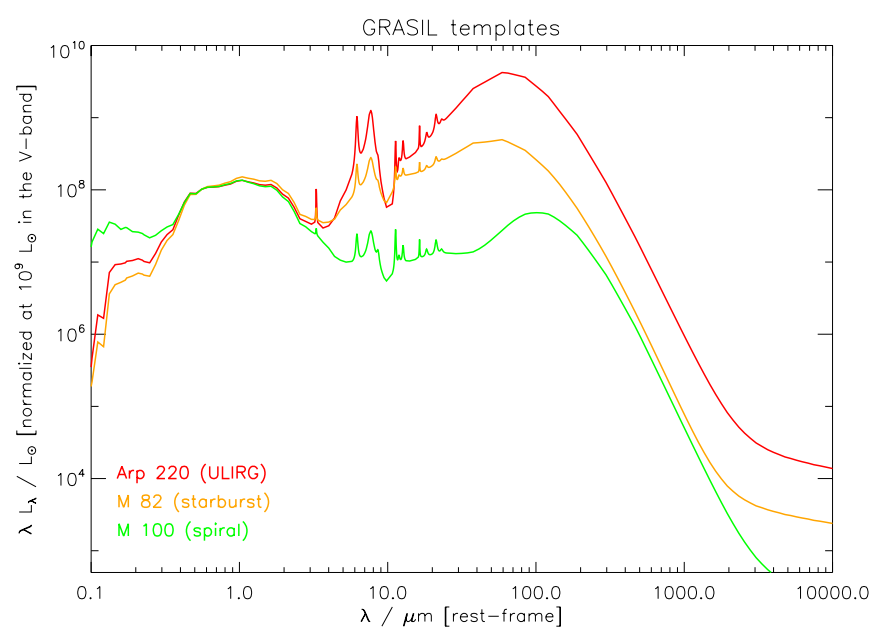

Figure 12. Ultraviolet to millimeter spectral energy distributions of Arp 220 (ULIRG), M 82 (low-luminosity starburst), and M 100 (spiral galaxy) from Silva et al. (1998), normalized in the $V$ band to emphasize their different optical-toinfrared ratios.

(A color version of this figure is available in the online journal.)

infrared-to-optical ratios typical of very dust-obscured ULIRGs, we would strongly overestimate the EBL at $870 \mu \mathrm{m}$ (Appendix A). The second assumption is that the empirical relations between infrared luminosities and line emission, calibrated for low-luminosity galaxies only in the local universe for obvious observational limitations, still hold for low-luminosity galaxies out to $z \simeq 5$. With this in mind, we have discussed how our results depend on the adopted $L_{\mathrm{IR}}-L_{\mathrm{CO}}^{\prime}$ correlation. To compute the luminosities of $\mathrm{CO}$ lines with upper level $J \geqslant 2$ from the $\mathrm{CO}(1-0)$ line luminosity, we rely on empirical $\mathrm{CO}$ SLEDs, which are a tracer of the excitation state of the gas. Since this is a particularly unknown for high-redshift low-luminosity galaxies, we discuss predicted $\mathrm{CO}$ line luminosities assuming two extreme CO SLEDs-Milky Way and M82-which should bracket all possible $\mathrm{CO}$ excitations in these galaxies (assuming that star formation is the only excitation source, i.e., no AGNs).
Considering these uncertainties, our predictions are the best possible based on the current empirical knowledge of star-forming galaxies.

A possible caveat of the optical selection of our sample is that we may be missing very optically faint dust-obscured sources, which would not be included in the Coe et al. (2006) optical catalog but could be bright in the (sub-)millimeter. This is most relevant for galaxies with $z>2$, where our optical catalog becomes incomplete (Figure 1). However, based on how well our predicted EBL and number counts at $870 \mu \mathrm{m}$ agree with observations, we conclude that such sources are probably not dominant. If very dust-obscured sources that are not included in our catalog exist in the considered field, our optically based predictions can be considered a lower limit for the number of detections in a (sub-)millimeter survey.

Barring cosmic variance, our predictions should provide a statistical representation of an arbitrarily chosen region on the sky, and can be used to plan deep field observations in the (sub-)millimeter regime. We have illustrated the usefulness of these predictions by estimating the expected number of continuum and line detections in a $500 \mathrm{hr}$ deep survey of the Hubble UDF with ALMA. For example, at $230 \mathrm{GHz}$ (ALMA band 6), considering the required number of pointings necessary to cover the total area of the UDF (given the primary beam size), we expect a continuum sensitivity of $5.1 \mu \mathrm{Jy}$ (computed using the ASC, assuming a bandwidth of $8 \mathrm{GHz}$ ). According to our predictions, this would yield a total of $\sim 6003 \sigma$ detections of galaxies distributed uniformly in redshift, with SFRs down to $1 M_{\odot} \mathrm{yr}^{-1}$ and infrared luminosities down to $10^{10} L_{\odot}$. We also discuss the predicted number of $\mathrm{CO}$ line detections when performing a scan over the whole frequency range covered by each band in steps of $8 \mathrm{GHz}$ (the interval covered in each frequency setting). In $500 \mathrm{hr}$, the line sensitivity reached by such a frequency scan of each UDF pointing in band 6 is $84 \mu \mathrm{Jy}$ (assuming a $300 \mathrm{~km} \mathrm{~s}^{-1}$ bandwidth), which would yield between 19 and 137 detections, depending on the $\mathrm{CO}$ excitation.

The predictions presented in this paper will help to plan future (sub-)millimeter line and continuum deep field campaigns
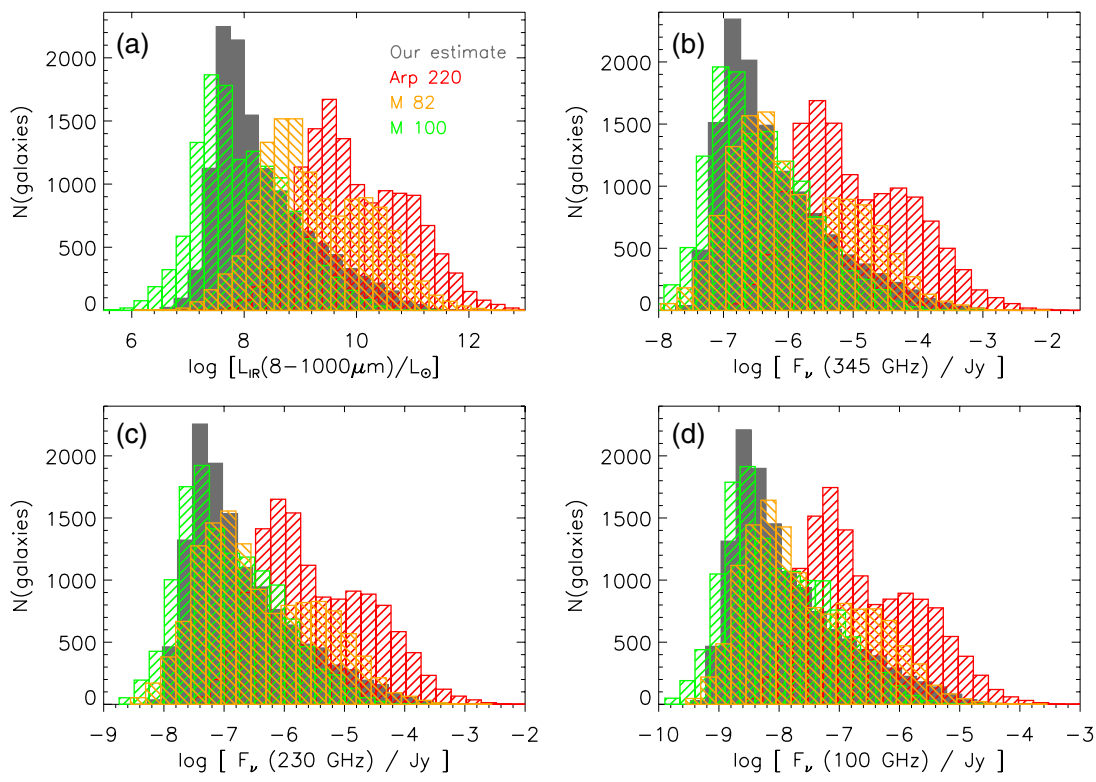

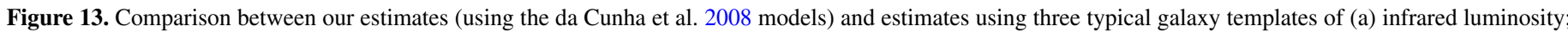
(b)-(d) distribution of continuum flux density at 345, 230, and $100 \mathrm{GHz}$ (ALMA bands 7, 6, 3; PdBI/NOEMA bands 4, 3, 1), respectively.

(A color version of this figure is available in the online journal.) 
Table 5

Redshift Ranges Where the $\mathrm{CO}$ and [C II] Lines are Observable as a Function of Band Frequency Range

\begin{tabular}{|c|c|c|c|c|c|c|c|c|c|}
\hline Band & $\begin{array}{l}\text { Frequency Range } \\
\qquad(\mathrm{GHz})\end{array}$ & $\begin{array}{c}\mathrm{CO}(1-0) \\
115.27 \mathrm{GHz}\end{array}$ & $\begin{array}{c}\mathrm{CO}(2-1) \\
230.54 \mathrm{GHz}\end{array}$ & $\begin{array}{c}\mathrm{CO}(3-2) \\
345.80 \mathrm{GHz}\end{array}$ & $\begin{array}{c}\mathrm{CO}(4-3) \\
461.04 \mathrm{GHz}\end{array}$ & $\begin{array}{c}\mathrm{CO}(5-4) \\
576.27 \mathrm{GHz}\end{array}$ & $\begin{array}{c}\mathrm{CO}(6-5) \\
691.47 \mathrm{GHz}\end{array}$ & $\begin{array}{c}\mathrm{CO}(7-6) \\
806.65 \mathrm{GHz}\end{array}$ & $\begin{array}{c}{\left[\mathrm{C}_{\text {III }}\right]} \\
1900.54 \mathrm{GHz}\end{array}$ \\
\hline JVLA $K$ & $18-26.5$ & $3.35-5.40$ & $7.70-11.8$ & & $\ldots$ & $\ldots$ & $\ldots$ & $\ldots$ & $\ldots$ \\
\hline JVLA $K a$ & $26.5-40$ & $1.88-3.35$ & $4.76-7.70$ & $7.65-12.0$ & $\ldots$ & $\ldots$ & $\ldots$ & $\ldots$ & $\ldots$ \\
\hline ALMA 1 & $31.3-45$ & $1.56-2.68$ & $4.12-6.37$ & $6.68-10.0$ & $9.24-13.7$ & $\ldots$ & $\ldots$ & $\ldots$ & $\ldots$ \\
\hline JVLA $Q$ & $40-50$ & $1.31-1.88$ & $3.61-4.76$ & $5.91-7.65$ & $8.22-10.5$ & $\ldots$ & $\ldots$ & $\ldots$ & $\ldots$ \\
\hline ALMA 2 & $67-90$ & $0.28-0.72$ & $1.56-2.44$ & $2.84-4.16$ & $4.12-5.88$ & $5.40-7.60$ & $6.68-9.32$ & $7.97-11.0$ & $\ldots$ \\
\hline ALMA 3 & $84-116$ & $0.00-0.37$ & $0.99-1.74$ & $1.98-3.12$ & $2.97-4.49$ & $3.97-5.86$ & $4.96-7.23$ & $5.95-8.60$ & $\ldots$ \\
\hline PdBI/NOEMA 1 & $80-116$ & $0.00-0.44$ & $0.99-1.88$ & $1.98-3.32$ & $2.97-4.76$ & $3.97-6.20$ & $4.96-7.64$ & $5.95-9.08$ & $\ldots$ \\
\hline ALMA 4 & $125-163$ & $\ldots$ & $0.41-0.84$ & $1.12-1.77$ & $1.83-2.69$ & $2.54-3.61$ & $3.24-4.53$ & $3.95-5.45$ & $\ldots$ \\
\hline PdBI/NOEMA 2 & $129-174$ & $\ldots$ & $0.32-0.79$ & $0.99-1.68$ & $1.66-2.57$ & $2.31-3.47$ & $2.97-4.36$ & $3.64-5.25$ & $\ldots$ \\
\hline ALMA 6 & $211-275$ & $\ldots$ & $0.00-0.09$ & $0.26-0.64$ & $0.68-1.19$ & $1.10-1.73$ & $1.51-2.28$ & $1.93-2.82$ & $5.91-8.01$ \\
\hline PdBI/NOEMA 3 & $201-267$ & $\ldots$ & $0.00-0.15$ & $0.30-0.72$ & $0.73-1.29$ & $1.16-1.87$ & $1.59-2.44$ & $2.02-3.01$ & $6.12-8.46$ \\
\hline ALMA 7 & $275-373$ & $\ldots$ & $\ldots$ & $0.00-0.26$ & $0.24-0.68$ & $0.55-1.10$ & $0.85-1.51$ & $1.16-1.93$ & $4.10-5.91$ \\
\hline PdBI/NOEMA 4 & $277-371$ & $\ldots$ & $\ldots$ & $0.00-0.25$ & $0.24-0.66$ & $0.55-1.08$ & $0.86-1.50$ & $1.17-1.91$ & $4.12-5.86$ \\
\hline ALMA 8 & $385-500$ & $\ldots$ & $\ldots$ & $\ldots$ & $0.00-0.20$ & $0.15-0.50$ & $0.38-0.80$ & $0.61-1.10$ & $2.80-3.94$ \\
\hline ALMA 9 & $602-710$ & $\ldots$ & $\ldots$ & $\ldots$ & $\ldots$ & $\ldots$ & $0.00-0.15$ & $0.12-0.34$ & $1.68-2.16$ \\
\hline ALMA 10 & $787-950$ & $\ldots$ & $\ldots$ & $\ldots$ & $\ldots$ & $\ldots$ & $\ldots$ & $0.00-0.03$ & $1.00-1.41$ \\
\hline
\end{tabular}

with new cutting-edge interferometers, and will also serve as a benchmark of our current empirical knowledge of dust continuum emission and $\mathrm{CO} /[\mathrm{C} \mathrm{II}]$ line emission, to which these future deep observations can be compared.

We thank the anonymous referee for comments that helped improve the quality and clarity of this paper. We also thank Vassilis Charmandaris and Eric Murphy for insightful comments on the manuscript. E.d.C. acknowledges funding through the ERC grant "Cosmic Dawn." R.D. acknowledges funding from Germany's national research center for aeronautics and space (DLR, project FKZ 50 OR 1104). Herschel is an ESA space observatory with science instruments provided by European-led Principal Investigator consortia and with important participation from NASA.

\section{APPENDIX A}

\section{COMPARISON WITH OTHER SED TEMPLATES}

For reference, we compare our predicted flux densities with those obtained using three typical local galaxy SEDs: a ULIRG, Arp 220, a low-luminosity starburst, M 82, and a typical spiral galaxy, M 100. We use template SEDs from Silva et al. (1998) that cover the full spectrum from ultraviolet to radio wavelengths. In Figure 12, we plot these SEDs normalized in the $V$ band to illustrate the large range in optical-to-infrared ratio spanned by these templates.

We $k$-correct and re-scale each template to fit the observed optical magnitudes of each galaxy in our sample, and from that we predict what the continuum fluxes in the ALMA bands would be if the observed galaxy SED was the same as the template.

In Figure 13, we compare the distribution of the infrared luminosity of the galaxies and the continuum flux in three ALMA bands when using our da Cunha et al. (2008) SED fits and when using these three templates. These distributions show the range of infrared luminosities and (sub-)millimeter fluxes of our observed galaxies if they all had optical-to-infrared ratios and infrared SEDs similar to Arp 220, M 82, and M 100, versus our predictions, which make an educated guess by comparing the observed UV/optical SED of each galaxy with a library of models with a wide range of optical-to-infrared ratios and infrared SED shapes. It is clear that, if we assumed that all observed galaxies had Arp 220-like SEDs, the typical infrared luminosities and predicted continuum fluxes would be typically two orders of magnitude higher. An M 82 template, with a similar infrared SED shape but higher optical-to-infrared ratio, still produces infrared luminosities about one order of magnitude higher than our da Cunha et al. (2008) SED fits, but the typical (sub-)millimeter continuum fluxes are similar to ours, except for part of the sample presenting an excess of fluxes compared to our estimates. The typical spiral template, M 100, has the higher optical-to-infrared ratios and cooler typical dust temperature, and predicts similar, if not slightly lower, typical infrared luminosities and submillimeter continuum fluxes than our fits.

As in Section 4.5, we can compare the integrated continuum emission over the whole UDF, which, divided by the area of the field, allows us to get an estimate of the EBL. The predicted continuum fluxes at $345 \mathrm{GHz}$ (ALMA 7) from our da Cunha et al. (2008) SED fits yield an EBL of $45.6 \mathrm{Jy} \mathrm{deg}^{-2}$, which is consistent with observations, as discussed in Section 4.5. When using fixed SED templates, we get $389 \mathrm{Jy} \mathrm{deg}^{-2}$ (Arp 220), $52.5 \mathrm{Jy} \mathrm{deg}^{-2}$ (M 82), and $9.32 \mathrm{Jy} \mathrm{deg}^{-2}$ (M 100), i.e., in the case of Arp 220 we get too much EBL at $345 \mathrm{GHz}$ by almost a factor of 10, while in the case of M 100 we tend to underestimate the EBL at $345 \mathrm{GHz}$ by about a factor of five.

\section{APPENDIX B}

\section{REDSHIFT RANGES WHERE CO AND [C II] ARE OBSERVABLE IN TYPICAL (SUB-)MILLIMETER BANDS}

For reference, we show in this appendix the redshift ranges with the $\mathrm{CO}$ lines $(1-0)$ to $(7-6)$ and the $[\mathrm{C}$ II] line are observable in the typical (sub-)millimeter bands from ALMA, JVLA, and PdBI/NOEMA (Figure 14 and Table 5).

\section{APPENDIX C}

\section{PREDICTED (SUB-)MILLIMETER FLUXES FOR EACH GALAXY IN OUR SAMPLE}

In this Appendix, we provide the predicted continuum and line fluxes for each galaxy in our sample. In Table 6, we list the predicted continuum fluxes (including confidence ranges) in the various (sub-)millimeter bands. In Table 7, we provide 
Table 6

Predicted Continuum Fluxes and Confidence Ranges for the First 10 Galaxies in Our Sample

\begin{tabular}{|c|c|c|c|c|c|c|c|c|c|c|c|c|}
\hline & & & & $\begin{array}{c}\log \left(F_{\nu} / \mathrm{Jy}\right) \\
38 \mathrm{GHz}\end{array}$ & $\begin{array}{c}\log \left(F_{v} / \mathrm{Jy}\right) \\
80 \mathrm{GHz}\end{array}$ & $\begin{array}{c}\log \left(F_{v} / \mathrm{Jy}\right) \\
100 \mathrm{GHz}\end{array}$ & $\begin{array}{l}\nu / \mathrm{Jy}) \\
\mathrm{GHz}\end{array}$ & $\begin{array}{c}\log \left(F_{v} / \mathrm{Jy}\right) \\
230 \mathrm{GHz}\end{array}$ & $\begin{array}{c}\log \left(F_{v} / \mathrm{Jy}\right) \\
345 \mathrm{GHz}\end{array}$ & $\begin{array}{c}\log \left(F_{v} / \mathrm{Jy}\right) \\
430 \mathrm{GHz}\end{array}$ & $\begin{array}{c}\log \left(F_{v} / \mathrm{Jy}\right) \\
660 \mathrm{GHz}\end{array}$ & $870 \mathrm{GHz}$ \\
\hline & & 7. & & & & & & & & & & \\
\hline & & $27 \cdot 40 \cdot 45$ & & & & & & & & & & \\
\hline & $3 \cdot 32 \cdot 3917$ & $27: 49: 45.20$ & 281 & & & & & & & & & \\
\hline & $3.32 \cdot 3911$ & $27: 49: 44.90$ & 791 & & & & & & & & & \\
\hline & $03: 3$ & $-27: 49: 44.01$ & 452 & & & & & & & & & -5 \\
\hline & $03: 3$ & $-27 \cdot 49 \cdot 2$ & & & & & & & & & & \\
\hline & $03: 32: 39.10$ & $-27: 49: 43.91$ & 0.69 & & & $-8.18_{-0.55}^{+0.74}$ & & & & & & \\
\hline & $03: 32: 39.52$ & $-27: 49: 43.20$ & 1.029 & $-8.62_{-0.10}^{+0.71}$ & $-8.28_{-0.71}^{+0.30}$ & $-8.06_{-0.66}^{+0.35}$ & $-7.62_{-0.63}^{+0.41}$ & $-6.93_{-0.60}^{+0.44}$ & $-6.39_{-0.60}^{+0.44}$ & $-6.18_{-0.60}^{+0.43}$ & $-5.68_{-0.65}^{+0.37}$ & $-5.49_{-0}^{+0.3}$ \\
\hline
\end{tabular}

Notes. The predicted fluxes are the median of the likelihood distribution computed as described in Section 3.4, and the confidence ranges correspond to the 16th-84th percentile of the likelihood distribution. IDs are the same as in the Coe et al. (2006) catalog.

(This table is available in its entirety in a machine-readable form in the online journal. A portion is shown here for guidance regarding its form and content.)

Table 7

Predicted $\mathrm{CO}$ and $[\mathrm{C}$ II] Line Fluxes for the First 10 Galaxies in Our Sample

\begin{tabular}{|c|c|c|c|c|c|c|c|c|c|}
\hline ID & $z_{\text {phot }}$ & $\begin{array}{c}\log \left(S_{\nu} / \mathrm{Jy}\right) \\
\operatorname{CO}(1-0)\end{array}$ & $\begin{array}{c}\log \left(S_{v} / \mathrm{Jy}\right) \\
\mathrm{CO}(2-1) \\
\mathrm{MW} / \mathrm{M} 82\end{array}$ & $\begin{array}{c}\log \left(S_{v} / \mathrm{Jy}\right) \\
\mathrm{CO}(3-2) \\
\mathrm{MW} / \mathrm{M} 82\end{array}$ & $\begin{array}{c}\log \left(S_{v} / \mathrm{Jy}\right) \\
\mathrm{CO}(4-3) \\
\mathrm{MW} / \mathrm{M} 82\end{array}$ & $\begin{array}{c}\log \left(S_{v} / \mathrm{Jy}\right) \\
\mathrm{CO}(5-4) \\
\text { MW/M82 }\end{array}$ & $\begin{array}{c}\log \left(S_{v} / \mathrm{Jy}\right) \\
\mathrm{CO}(6-5) \\
\mathrm{MW} / \mathrm{M} 82\end{array}$ & $\begin{array}{c}\log \left(S_{v} / \mathrm{Jy}\right) \\
\mathrm{CO}(7-6) \\
\mathrm{MW} / \mathrm{M} 82\end{array}$ & $\begin{array}{c}\log \left(S_{v} / \mathrm{Jy}\right) \\
{\left[\mathrm{C}_{\mathrm{II}}\right]}\end{array}$ \\
\hline 1 & 0.472 & -4.58 & $-4.28 /-3.99$ & $-4.19 /-3.66$ & $-4.14 /-3.45$ & $-4.27 /-3.31$ & $-4.50 /-3.24$ & $-4.63 /-3.30$ & -2.36 \\
\hline 2 & 2.703 & -6.64 & $-6.34 /-6.04$ & $-6.25 /-5.72$ & $-6.20 /-5.50$ & $-6.32 /-5.37$ & $-6.56 /-5.30$ & $-6.69 /-5.36$ & -4.50 \\
\hline 3 & 1.281 & -7.23 & $-6.93 /-6.64$ & $-6.84 /-6.31$ & $-6.79 /-6.10$ & $-6.92 /-5.96$ & $-7.16 /-5.90$ & $-7.28 /-5.96$ & -5.26 \\
\hline 4 & 3.791 & -6.66 & $-6.35 /-6.06$ & $-6.26 /-5.73$ & $-6.21 /-5.52$ & $-6.34 /-5.38$ & $-6.58 /-5.32$ & $-6.70 /-5.38$ & -4.49 \\
\hline 5 & 0.452 & -5.83 & $-5.52 /-5.23$ & $-5.44 /-4.91$ & $-5.38 /-4.69$ & $-5.51 /-4.55$ & $-5.75 /-4.49$ & $-5.88 /-4.55$ & -3.78 \\
\hline 6 & 2.769 & -6.95 & $-6.65 /-6.36$ & $-6.56 /-6.03$ & $-6.51 /-5.82$ & $-6.64 /-5.68$ & $-6.87 /-5.62$ & $-7.00 /-5.68$ & -4.86 \\
\hline 7 & 1.653 & -5.73 & $-5.42 /-5.13$ & $-5.33 /-4.80$ & $-5.28 /-4.59$ & $-5.41 /-4.45$ & $-5.65 /-4.39$ & $-5.77 /-4.45$ & -3.52 \\
\hline 8 & 0.636 & -3.94 & $-3.64 /-3.35$ & $-3.55 /-3.02$ & $-3.50 /-2.81$ & $-3.63 /-2.67$ & $-3.86 /-2.61$ & $-3.99 /-2.66$ & -1.60 \\
\hline 9 & 0.695 & -6.41 & $-6.11 /-5.82$ & $-6.02 /-5.49$ & $-5.97 /-5.28$ & $-6.10 /-5.14$ & $-6.33 /-5.08$ & $-6.46 /-5.14$ & -4.40 \\
\hline 10 & 1.029 & -6.27 & $-5.96 /-5.67$ & $-5.88 /-5.35$ & $-5.82 /-5.52$ & $-5.95 /-4.99$ & $-6.19 /-4.93$ & $-6.31 /-3.98$ & -4.18 \\
\hline
\end{tabular}

Notes. For the CO lines, we include the flux prediction using a Milky Way CO SLED (MW) and an M82 center SLED (M82), as described in Section 4.7.1. IDs are the same as in the Coe et al. (2006) catalog.

(This table is available in its entirety in a machine-readable form in the online journal. A portion is shown here for guidance regarding its form and content.)

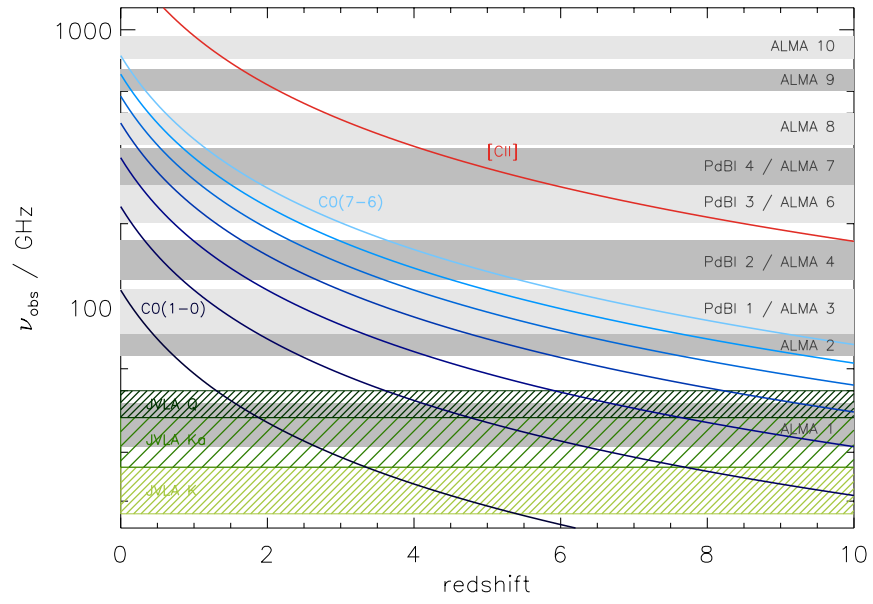

Figure 14. Observed frequency of the $\mathrm{CO}$ lines $\mathrm{CO}(1-0)$ to $\mathrm{CO}(7-6)$ (from darker blue to lighter blue lines) and [C II] $158 \mu \mathrm{m}$ (red line) as a function of redshift. The gray-shaded and green-shaded areas show the frequency ranges sampled by the ALMA, JVLA, and PdBI/NOEMA bands considered in this paper.

(A color version of this figure is available in the online journal.) the predicted fluxes of the CO lines (using both Milky Way and M82 SLEDs) and of the [C II] line.

\section{REFERENCES}

Bell, E. F. 2003, ApJ, 586, 794

Bell, E. F., McIntosh, D. H., Katz, N., \& Weinberg, M. D. 2003, ApJS, 149, 289 Borys, C., Chapman, S., Halpern, M., \& Scott, D. 2003, MNRAS, 344, 385 Boselli, A., Gavazzi, G., Lequeux, J., \& Pierini, D. 2002, A\&A, 385, 454 Bouwens, R. J., Illingworth, G. D., Oesch, P. A., et al. 2010, ApJL, 709, L133 Brauher, J. R., Dale, D. A., \& Helou, G. 2008, ApJS, 178, 280

Bruzual, G., \& Charlot, S. 2003, MNRAS, 344, 1000 Charlot, S., \& Fall, S. M. 2000, ApJ, 539, 718

Coe, D., Benítez, N., Sánchez, S. F., et al. 2006, AJ, 132, 926

Combes, F., Maoli, R., \& Omont, A. 1999, A\&A, 345, 369

Condon, J. J. 1992, ARA\&A, 30, 575

Coppin, K., Chapin, E. L., Mortier, A. M. J., et al. 2006, MNRAS, 372, 1621 Cox, P., Krips, M., Neri, R., et al. 2011, ApJ, 740, 63 da Cunha, E., Charlot, S., \& Elbaz, D. 2008, MNRAS, 388, 1595 da Cunha, E., Charmandaris, V., Díaz-Santos, T., et al. 2010, A\&A, 523, A78 Daddi, E., Bournaud, F., Walter, F., et al. 2010a, ApJ, 713, 686 Daddi, E., Dannerbauer, H., Elbaz, D., et al. 2008, ApJL, 673, L21 Daddi, E., Dannerbauer, H., Krips, M., et al. 2009a, ApJL, 695, L176

Daddi, E., Dannerbauer, H., Stern, D., et al. 2009b, ApJ, 694, 1517 Daddi, E., Dickinson, M., Chary, R., et al. 2005, ApJL, 631, L13

Daddi, E., Dickinson, M., Morrison, G., et al. 2007, ApJ, 670, 156

Daddi, E., Elbaz, D., Walter, F., et al. 2010b, ApJL, 714, L118 
Dale, D. A., \& Helou, G. 2002, ApJ, 576, 159

de Looze, I., Baes, M., Bendo, G. J., Cortese, L., \& Fritz, J. 2011, MNRAS, 416,2712

Dunlop, J. S. 2011, arXiv:1108.5679

Elbaz, D., Dickinson, M., Hwang, H. S., et al. 2011, A\&A, 533, A119

Fixsen, D. J., Dwek, E., Mather, J. C., Bennett, C. L., \& Shafer, R. A. 1998, ApJ, 508,123

Geach, J. E., Smail, I., Moran, S. M., et al. 2011, ApJL, 730, L19

Genzel, R., Tacconi, L. J., Gracia-Carpio, J., et al. 2010, MNRAS, 407, 2091

Graciá-Carpio, J., Sturm, E., Hailey-Dunsheath, S., et al. 2011, ApJL, 728, L7

Helou, G., Soifer, B. T., \& Rowan-Robinson, M. 1985, ApJL, 298, L7

Hopkins, A. M., \& Beacom, J. F. 2006, ApJ, 651, 142

Ilbert, O., Salvato, M., Le Floc'h, E., et al. 2010, ApJ, 709, 644

Karim, A., Schinnerer, E., Martínez-Sansigre, A., et al. 2011, ApJ, 730, 61

Knudsen, K. K., van der Werf, P. P., \& Kneib, J.-P. 2008, MNRAS, 384, 1611

Lagos, C. D. P., Baugh, C. M., Lacey, C. G., et al. 2011, MNRAS, 418, 1649

Le Fèvre, O., Vettolani, G., Garilli, B., et al. 2005, A\&A, 439, 845

Lilly, S. J., Le Fevre, O., Hammer, F., \& Crampton, D. 1996, ApJL, 460, L1

Lilly, S. J., Le Fèvre, O., Renzini, A., et al. 2007, ApJS, 172, 70

Madau, P., Ferguson, H. C., Dickinson, M. E., et al. 1996, MNRAS, 283, 1388

Magdis, G. E., Elbaz, D., Dickinson, M., et al. 2011, A\&A, 534, A15

Obreschkow, D., Croton, D., De Lucia, G., Khochfar, S., \& Rawlings, S. 2009a, ApJ, 698, 1467

Obreschkow, D., Heywood, I., Klöckner, H.-R., \& Rawlings, S. 2009b, ApJ, 702,1321

Obreschkow, D., Heywood, I., \& Rawlings, S. 2011, ApJ, 743, 84

Oliver, S. J., Wang, L., Smith, A. J., et al. 2010, A\&A, 518, L21
Power, C., Baugh, C. M., \& Lacey, C. G. 2010, MNRAS, 406, 43 Puget, J.-L., Abergel, A., Bernard, J.-P., et al. 1996, A\&A, 308, L5 Reddy, N. A., Steidel, C. C., Fadda, D., et al. 2006, ApJ, 644, 792

Riechers, D. A., Walter, F., Carilli, C. L., et al. 2006, ApJ, 650, 604 Rodighiero, G., Daddi, E., Baronchelli, I., et al. 2011, ApJL, 739, L40 Sargent, M. T., Béthermin, M., Daddi, E., \& Elbaz, D. 2012, ApJL, 747, L31 Sargent, M. T., Schinnerer, E., Murphy, E., et al. 2010, ApJL, 714, L190 Scott, S. E., Dunlop, J. S., \& Serjeant, S. 2006, MNRAS, 370, 1057

Scott, S. E., Fox, M. J., Dunlop, J. S., et al. 2002, MNRAS, 331, 817 Silva, L., Granato, G. L., Bressan, A., \& Danese, L. 1998, ApJ, 509, 103

Smail, I., Ivison, R. J., Blain, A. W., \& Kneib, J.-P. 2002, MNRAS, 331, 495

Smolčić, V., Schinnerer, E., Zamorani, G., et al. 2009, ApJ, 690, 610

Solomon, P. M., \& Vanden Bout, P. A. 2005, ARA\&A, 43, 677

Springel, V., White, S. D. M., Jenkins, A., et al. 2005, Natur, 435, 629

Stacey, G. J., Geis, N., Genzel, R., et al. 1991, ApJ, 373, 423

Stacey, G. J., Hailey-Dunsheath, S., Ferkinhoff, C., et al. 2010, ApJ, 724,957

Steidel, C. C., Adelberger, K. L., Shapley, A. E., et al. 2003, ApJ, 592, 728

Tacconi, L. J., Genzel, R., Neri, R., et al. 2010, Natur, 463, 781

Walter, F., Carilli, C., \& Daddi, E. 2011, arXiv:1101.4022

Walter, F., Decarli, R., Carilli, C., et al. 2012, Natur, 486, 233

Weiß, A., Kovács, A., Coppin, K., et al. 2009, ApJ, 707, 1201

Weiss, A., Downes, D., Walter, F., \& Henkel, C. 2007, in ASP Conf. Ser. 375, From Z-Machines to ALMA: (Sub)Millimeter Spectroscopy of Galaxies, ed. A. J. Baker, J. Glenn, A. I. Harris, J. G. Mangum, \& M. S. Yun (San Francisco, CA: ASP), 25

Wuyts, S., Labbé, I., Schreiber, N. M. F., et al. 2008, ApJ, 682, 985

Yun, M. S., Reddy, N. A., \& Condon, J. J. 2001, ApJ, 554, 803 